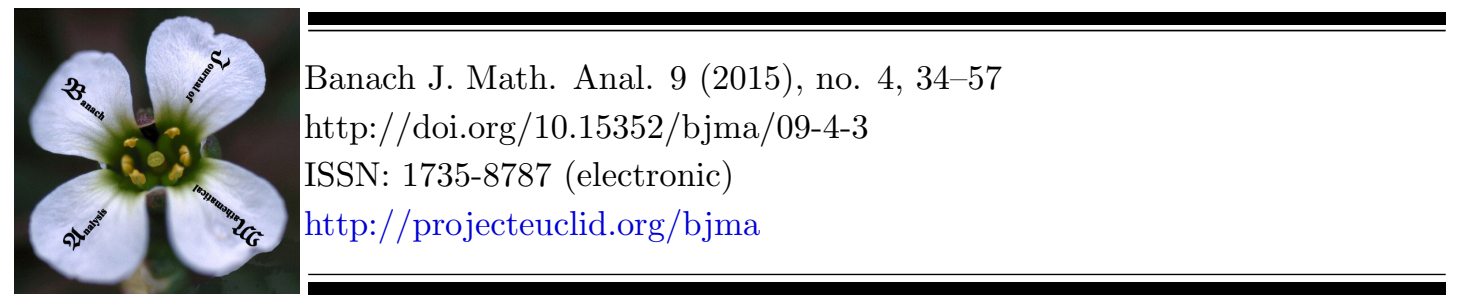

\title{
LIPSCHITZ GROTHENDIECK-INTEGRAL OPERATORS
}

\author{
M. G. CABRERA-PADILLA AND A. JIMÉNEZ-VARGAS* \\ Communicated by K. Jarosz
}

\begin{abstract}
Let $X$ be a pointed metric space and let $E$ be a Banach space. It is known that the Lipschitz $\operatorname{space}^{\operatorname{Lip}_{0}}\left(X, E^{*}\right)$ is isometrically isomorphic to $\left(\mathcal{F}(X) \widehat{\otimes}_{\pi} E\right)^{*}$, the dual of the projective tensor product of the Lipschitz-free space $\mathcal{F}(X)$ and $E$. Since the injective norm $\varepsilon$ on $\mathcal{F}(X) \otimes E$ is smaller than the projective norm $\pi$, we study Lipschitz Grothendieck-integral operators which are exactly those elements in $\operatorname{Lip}_{0}\left(X, E^{*}\right)$ which correspond to the elements of $\left(\mathcal{F}(X) \widehat{\otimes}_{\varepsilon} E\right)^{*}$, the dual of the injective tensor product of $\mathcal{F}(X)$ and $E$.
\end{abstract}

\section{INTRODUCTION}

Let $X$ be a pointed metric space with a base point denoted by 0 , and let $E$ be a Banach space over $\mathbb{K}$. The Lipschitz $\operatorname{space}^{\operatorname{Lip}_{0}}(X, E)$ is the Banach space of all Lipschitz maps $f$ from $X$ into $E$ that vanish at 0 , under the Lipschitz norm defined by

$$
\operatorname{Lip}(f)=\sup \left\{\frac{\|f(x)-f(y)\|}{d(x, y)}: x, y \in X, x \neq y\right\} .
$$

The elements of $\operatorname{Lip}_{0}(X, E)$ are referred as Lipschitz operators. $\operatorname{Lip}_{0}(X, \mathbb{K})$ is known as the Lipschitz dual of $X$ and denoted by $X^{\#}$. The closed linear subspace of the dual of $X^{\#}$ spanned by the point evaluation functionals $\delta_{x}$ on $X^{\#}$ with $x \in X$ is a predual of $X^{\#}$ called the Lipschitz-free space over $X$ and denoted $\mathcal{F}(X)$ by Godefroy and Kalton in [8]. The Weaver's book [13] contains a complete study

Date: Received: Nov. 9, 2014; Accepted: Dec. 11, 2014.

* Corresponding author.

2010 Mathematics Subject Classification. Primary 46B28; Secondary 26A16, 46E15, 47L20.

Key words and phrases. Lipschitz map, tensor product, integral operator, Pietsch integral operator, nuclear operator. 
of $\operatorname{Lip}_{0}(X)$ and its predual $\mathcal{F}(X)$ which is called the Arens-Eells space of $X$ and denoted by $\mathbb{E}(X)$ there.

Let $E$ and $F$ be Banach spaces. As usual, $B_{E}$ stands for the closed unit ball of $E, E^{*}$ for the topological dual of $E$ and $\kappa_{E}$ for the canonical injection from $E$ into $E^{* *}$. We denote by $E \widehat{\otimes}_{\varepsilon} F$ the completion of the algebraic tensor product $E \otimes F$ in the injective norm defined by

$$
\varepsilon\left(\sum_{i=1}^{n} x_{i} \otimes y_{i}\right)=\sup \left\{\left|\sum_{i=1}^{n} f\left(x_{i}\right) g\left(y_{i}\right)\right|: f \in B_{E^{*}}, g \in B_{F^{*}}\right\},
$$

and by $E \widehat{\otimes}_{\pi} F$ the completion of $E \otimes F$ in the projective norm defined by

$$
\pi\left(\sum_{i=1}^{n} x_{i} \otimes y_{i}\right)=\inf \left\{\sum_{j=1}^{k}\left\|x_{j}^{\prime}\right\|\left\|y_{j}^{\prime}\right\|: \sum_{j=1}^{k} x_{j}^{\prime} \otimes y_{j}^{\prime}=\sum_{i=1}^{n} x_{i} \otimes y_{i}\right\} .
$$

The space $\operatorname{Lip}_{0}\left(X, E^{*}\right)$ is isometrically isomorphic to $\left(\mathcal{F}(X) \widehat{\otimes}_{\pi} E\right)^{*}$, via the map $f \mapsto \Gamma(f)$ given by

$$
\left\langle\Gamma(f), \delta_{x} \otimes e\right\rangle=\langle f(x), e\rangle
$$

for $f \in \operatorname{Lip}_{0}\left(X, E^{*}\right)$ and $\delta_{x} \otimes e \in \mathcal{F}(X) \otimes E$ (essentially, [10, Theorems 4.1 and 5.8]). Since $\varepsilon \leq \pi$ on $\mathcal{F}(X) \otimes E$, we are interested in determining those Lipschitz operators $f$ in $\operatorname{Lip}_{0}\left(X, E^{*}\right)$ which correspond to the elements of the space $\left(\mathcal{F}(X) \widehat{\otimes}_{\varepsilon} E\right)^{*}$. We will prove that those Lipschitz operators are exactly Lipschitz Grothendieck-integral operators from $X$ into $E^{*}$, defined as follows.

Definition 1. Let $X$ be a pointed metric space and let $E$ be a Banach space. A Lipschitz operator $f \in \operatorname{Lip}_{0}(X, E)$ is called a Lipschitz Grothendieck-integral (G-integral for short) operator if there exist a finite measure space $(\Omega, \Sigma, \mu)$, a bounded linear operator $A \in \mathcal{L}\left(L_{1}(\mu), E^{* *}\right)$ and a Lipschitz operator $b \in$ $\operatorname{Lip}_{0}\left(X, L_{\infty}(\mu)\right)$ such that the following diagram commutes:

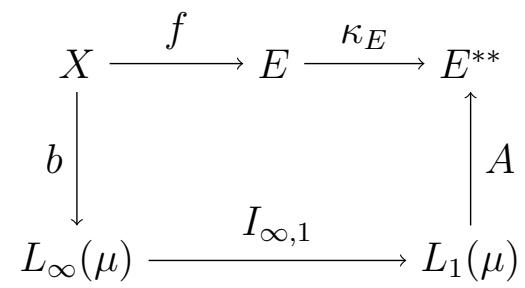

where $I_{\infty, 1}: L_{\infty}(\mu) \rightarrow L_{1}(\mu)$ is the formal inclusion operator. The triple $(A, b, \mu)$ is called a Lipschitz G-integral factorization of $f$. The Lipschitz G-integral norm of $f$ is defined by $\operatorname{Lip}_{G I}(f)=\inf \|A\| \operatorname{Lip}(b) \mu(\Omega)$, where the infimum is taken over all Lipschitz G-integral factorizations of $f$. The set of all Lipschitz G-integral operators from $X$ to $E$ will be denoted by $\operatorname{Lip}_{0 G I}(X, E)$. Multiplying $A$ or $b$ by a constant, we can assume that $\mu$ is a probability measure.

In 2009, Farmer and Johnson started in [7] the study of Lipschitz p-summing operators between metric spaces, a nonlinear generalization of $p$-summing operators. From then, some Lipschitz versions of different classes of bounded linear operators have been investigated as, for example, Lipschitz $(p, r, s)$-summing $((q, p)$ mixing, completely $(q, p)$-mixing) operators by Chávez-Domínguez in $[1,2,3]$, 
Lipschitz $p$-nuclear ( $p$-integral, $p$-dominated) operators by Chen and Zheng in [4, 5], Lipschitz strongly p-summing operators by Saadi in [12], and Lipschitz compact (weakly compact, finite-rank, approximable) operators by Sepulcre, Villegas and the second-named author in [9].

This paper is devoted to the study of Lipschitz G-integral operators from $X$ to $E$ and their relationships with other classes of Lipschitz operators as Lipschitz finite-rank operators, Lipschitz Pietsch-integral operators and Lipschitz nuclear operators.

Before going to describe the contents of this paper, we recall the following concepts.

Definition 2. Let $X$ be a pointed metric space and let $E$ be a Banach space. For any $g \in X^{\#}$ and $e \in E$, let $g \cdot e$ be the map from $X$ to $E$ defined by $(g \cdot e)(x)=g(x) e$ for all $x \in X$. Let $A(X, E)$ be a linear subspace of $\operatorname{Lip}_{0}(X, E)$ equipped with a norm $\|\cdot\|_{A}$. Consider the following conditions:

(i) $\operatorname{Lip}(f) \leq\|f\|_{A}$ for every $f \in A(X, E)$.

(ii) $\left(A(X, E),\|\cdot\|_{A}\right)$ is a Banach space.

(iii) If $g \in X^{\#}$ and $e \in E$, then $g \cdot e \in A(X, E)$ and $\|g \cdot e\|_{A} \leq \operatorname{Lip}(g)\|e\|$.

(iv) The ideal property: Let $Y$ be a pointed metric space and let $F$ be a Banach space. If $h \in \operatorname{Lip}_{0}(Y, X), f \in A(X, E)$ and $T \in \mathcal{L}(E, F)$, then $T f h \in A(Y, F)$ and $\|T f h\|_{A} \leq\|T\|\|f\|_{A} \operatorname{Lip}(h)$.

It is said that $\left(A(X, E),\|\cdot\|_{A}\right)$ is a Banach space (Banach ideal) of Lipschitz operators if it satisfies the properties (i), (ii) and (iii) (respectively, (ii), (iii) and (iv)).

Section 1 gathers the main properties of Lipschitz finite-rank operators, some preliminary results about the Lipschitz-free space $\mathcal{F}(X)$ and an integral description for the elements of $\left(\mathcal{F}(X) \widehat{\otimes}_{\varepsilon} E\right)^{*}$.

In Section 2, we show that $\left(\operatorname{Lip}_{0 G I}(X, E), \operatorname{Lip}_{G I}\right)$ is a Banach space and a Banach ideal of Lipschitz operators. Our main result in Section 2 characterizes Lipschitz G-integral operators from $X$ to $E$ as those Lipschitz operators $f$ in $\operatorname{Lip}_{0}(X, E)$ whose associate linear functionals $\Gamma\left(\kappa_{E} f\right)$ on $\mathcal{F}(X) \otimes E^{*}$ are continuous on $\mathcal{F}(X) \widehat{\otimes}_{\varepsilon} E^{*}$. Furthermore, $\operatorname{Lip}_{G I}(f)=\left\|\Gamma\left(\kappa_{E} f\right)\right\|$. We also show that the space $L_{\infty}(\mu)$ in Definition 1 can be replaced by the space $C_{w^{*}}\left(B_{X^{\#}}\right)$ of all weak* continuous functions from $B_{X^{\#}}$ into $\mathbb{K}$.

Let us recall that the Lipschitz transpose operator of a Lipschitz operator $f \in \operatorname{Lip}_{0}(X, E)$ is the bounded linear operator $f^{t}: E^{*} \rightarrow X^{\#}$ defined by $f^{t}(\phi)=$ $\phi \circ f$ for all $\phi \in E^{*}$. Furthermore, $\left\|f^{t}\right\|=\operatorname{Lip}(f)$ and $f^{t}=Q_{X}^{-1}\left(T_{f}\right)^{*}$, where $Q_{X}: X^{\#} \rightarrow \mathcal{F}(X)^{*}$ is the canonical isometric isomorphism and $T_{f}: \mathcal{F}(X) \rightarrow E$ is the linearization of $f$ provided by a known result of Weaver [13, Theorem 2.2.4]. We will prove that a Lipschitz operator $f \in \operatorname{Lip}_{0}(X, E)$ is Lipschitz G-integral if and only if its Lipschitz transpose operator $f^{t}$ (or its linearization $T_{f}$ ) is an integral linear operator from $E^{*}$ into $X^{\#}$ (respectively, from $\mathcal{F}(X)$ into $E$ ), in whose case $\operatorname{Lip}_{G I}(f)$ coincides with the integral norms of $f^{t}$ and $T_{f}$.

Section 3 deals with a subclass of Lipschitz G-integral operators from $X$ to $E$ called Lipschitz Pietsch-integral (P-integral for short) operators which are defined 
if we require to factor the operator $f: X \rightarrow E$ rather than $\kappa_{E} f$ in Definition 1. We prove that the set $\operatorname{Lip}_{0 P I}(X, E)$ of such operators is a Banach space (respectively, Banach ideal) of Lipschitz operators under the so-called Lipschitz P-integral norm $\operatorname{Lip}_{P I}$. When $E$ is a dual Banach space, we show that both spaces $\operatorname{Lip}_{0 G I}(X, E)$ and $\operatorname{Lip}_{0 P I}(X, E)$ coincide and use this fact to answer the question which motivated this paper by establishing that a Lipschitz operator $f \in$ $\operatorname{Lip}_{0}\left(X, E^{*}\right)$ is Lipschitz G-integral if and only if the associate linear functional $\Gamma(f)$ on $\mathcal{F}(X) \otimes E$ is continuous on $\mathcal{F}(X) \widehat{\otimes}_{\varepsilon} E$. Moreover the mapping $f \mapsto \Gamma(f)$ is an isometric isomorphism from $\left(\operatorname{Lip}_{0 G I}\left(X, E^{*}\right), \operatorname{Lip}_{G I}\right)$ onto $\left(\mathcal{F}(X) \widehat{\otimes}_{\varepsilon} E\right)^{*}$.

Section 4 is devoted to the set $\operatorname{Lip}_{0 N}(X, E)$ of Lipschitz nuclear operators from $X$ to $E$. We prove that $\operatorname{Lip}_{0 N}(X, E)$ is a Banach space (respectively, Banach ideal) of Lipschitz operators with the so-called Lipschitz nuclear norm $\operatorname{Lip}_{N}$. In fact, every element in $\operatorname{Lip}_{0 N}(X, E)$ is the $\operatorname{Lip}_{N^{-}}$-limit of a sequence of Lipschitz finiterank operators from $X$ to $E$. We also prove that $\operatorname{Lip}_{0 N}(X, E) \subset \operatorname{Lip}_{0 P I}(X, E)$ and $\operatorname{Lip}_{P I}(f) \leq \operatorname{Lip}_{N}(f)$ for all $f \in \operatorname{Lip}_{0 N}(X, E)$. Moreover, the converse inclusion and inequality hold when $E$ has the Radon-Nikodým property. Finally, it is shown that a Lipschitz operator $f \in \operatorname{Lip}_{0}(X, E)$ is a Lipschitz G-integral operator if and only if $f$ is the limit in the Lipschitz weak operator topology on $\operatorname{Lip}_{0}(X, E)$ of a $\operatorname{Lip}_{N}$-bounded net of Lipschitz nuclear operators (respectively, Lipschitz finite-rank operators) from $X$ to $E$.

\section{Preliminaries}

Throughout this paper, if $X$ and $Y$ are pointed metric spaces, we will denote by $\operatorname{Lip}_{0}(X, Y)$ the set of all base-point preserving Lipschitz maps from $X$ to $Y$. If $E$ and $F$ are Banach spaces, $\mathcal{L}(E, F)$ will stand for the Banach space of all bounded linear operators from $E$ into $F$ endowed with the usual norm.

An element $f \in \operatorname{Lip}_{0}(X, E)$ is said to be a Lipschitz finite-rank operator if the linear subspace of $E$ generated by $f(X), \operatorname{lin}(f(X))$, is finite dimensional in whose case the rank of $f$, denoted by $\operatorname{rank}(f)$, is defined as the dimension of $\operatorname{lin}(f(X))$. We denote by $\operatorname{Lip}_{0 F}(X, E)$ the set of all Lipschitz finite-rank operators from $X$ into $E$.

We gather some properties of those Lipschitz operators in the following easy result.

Proposition 1.1. Let $X$ be a pointed metric space and be $E$ a Banach space.

(i) $\operatorname{Lip}_{0 F}(X, E)$ is a linear subspace of $\operatorname{Lip}_{0}(X, E)$.

(ii) If $g \in X^{\#}$ and $e \in E$, then $g \cdot e \in \operatorname{Lip}_{0 F}(X, E)$ with $\operatorname{Lip}(g \cdot e)=\operatorname{Lip}(g)\|e\|$. Moreover, $\operatorname{rank}(g \cdot e)=1$ if $g \neq 0$ and $e \neq 0$.

(iii) Every element $f \in \operatorname{Lip}_{0 F}(X, E)$ has a representation in the form $f=$ $\sum_{i=1}^{n} g_{i} \cdot e_{i}$ where $n=\operatorname{rank}(f), g_{1}, \ldots, g_{n} \in X^{\#}$ and $e_{1}, \ldots, e_{n} \in E$.

(iv) If $f=\sum_{i=1}^{n} g_{i} \cdot e_{i}$ as in (iii), then $f^{t}: E^{*} \rightarrow X^{\#}$ has finite rank and $f^{t}=\sum_{i=1}^{n} \kappa_{E}\left(e_{i}\right) \cdot g_{i}$.

(v) If $Y$ is a pointed metric space, $F$ a Banach space, $h \in \operatorname{Lip}_{0}(Y, X)$, $f \in \operatorname{Lip}_{0}(X, E)$ and $T \in \mathcal{L}(E, F)$, then $T f h \in \operatorname{Lip}_{0 F}(Y, F)$ and $T f h=$ $\sum_{i=1}^{n}\left(g_{i} \circ h\right) \cdot T\left(e_{i}\right)$. 
We now recall some properties of the Lipschitz-free space $\mathcal{F}(X)$ that will be needed later.

Theorem 1.2. [13, pp. 39-41],[9] Let $X$ be a pointed metric space and $E$ a Banach space.

(i) The dual of $\mathcal{F}(X)$ is isometrically isomorphic to $X^{\#}$, via the evaluation map $Q_{X}: X^{\#} \rightarrow \mathcal{F}(X)^{*}$ defined by $Q_{X}(g)(\gamma)=\gamma(g)$ for all $g \in X^{\#}$ and $\gamma \in \mathcal{F}(X)$. Moreover, on bounded subsets of $X^{\#}$, the weak ${ }^{*}$ topology coincides with the topology of pointwise convergence.

(ii) The Dirac map $\delta_{X}: x \mapsto \delta_{x}$ is an isometric embedding of $X$ into $\mathcal{F}(X)$.

(iii) For any $f \in \operatorname{Lip}_{0}(X, E)$, there is a unique $T_{f} \in \mathcal{L}(\mathcal{F}(X), E)$ such that $T_{f} \circ \delta_{X}=f$. Furthermore, $\left\|T_{f}\right\|=\operatorname{Lip}(f)$ and $\left(T_{f}\right)^{*}=Q_{X} f^{t}$.

(iv) The space $\mathcal{F}(X)$ agrees with the space of all linear functionals $\gamma$ on $X^{\#}$ such that $\left.\gamma\right|_{B_{X} \#}$ is continuous in the topology of pointwise convergence.

Taking into account the density of the linear span of $\left\{\delta_{x}\right\}_{x \in X}$ in $\mathcal{F}(X)$, the following characterization of the elements of $(\mathcal{F}(X) \widehat{\otimes} E)^{*}$ can be deduced from the theory of tensor products of Banach spaces (see [11, Section 3.4]).

Theorem 1.3. Let $\varphi$ be a linear functional on $\mathcal{F}(X) \otimes E$. Then $\varphi$ is continuous on $\mathcal{F}(X) \widehat{\otimes}_{\varepsilon} E$ if and only if there exist a finite measure space $(\Omega, \Sigma, \mu)$, a bounded linear operator $A \in \mathcal{L}\left(E, L_{\infty}(\mu)\right)$ and a Lipschitz operator $b \in \operatorname{Lip}_{0}\left(X, L_{\infty}(\mu)\right)$ such that

$$
\left\langle\varphi, \delta_{x} \otimes e\right\rangle=\int_{\Omega} b(x) A(e) d \mu
$$

for all $x \in X$ and $e \in E$. Furthermore, the norm of $\varphi$ is given by $\|\varphi\|=$ $\inf \|A\| \operatorname{Lip}(b) \mu(\Omega)$, where the infimum is taken over all such factorizations of $\varphi$, and this infimum is attained. Multiplying $A$ or $b$ by a constant, we can assume that $\mu$ is a probability measure.

From now on, $\Phi: L_{\infty}(\mu) \rightarrow L_{1}(\mu)^{*}$ will denote the canonical isometric isomorphism given by

$$
\langle\Phi(t), s\rangle=\int_{\Omega} I_{\infty, 1}(t) s d \mu \quad\left(t \in L_{\infty}(\mu), s \in L_{1}(\mu)\right) .
$$

Note that, for any $\varphi \in L_{1}(\mu)^{*}$ and $s \in L_{1}(\mu)$, we have

$$
\langle\varphi, s\rangle=\left\langle\Phi\left(\Phi^{-1}(\varphi)\right), s\right\rangle=\int_{\Omega} I_{\infty, 1}\left(\Phi^{-1}(\varphi)\right) s d \mu .
$$

\section{Lipschitz GRothendiECK-INTEGRAL OPERATORS}

The question raised in the introduction can be posed to Lipschitz operators taking values in $E$ instead of $E^{*}$. Indeed, every Lipschitz operator $f \in \operatorname{Lip}_{0}(X, E)$ determines a Lipschitz operator $\kappa_{E} f \in \operatorname{Lip}_{0}\left(X, E^{* *}\right)$ which has an associate functional $\Gamma\left(\kappa_{E} f\right)$ in $\left(\mathcal{F}(X) \widehat{\otimes}_{\pi} E^{*}\right)^{*}$ defined by

$$
\left\langle\Gamma\left(\kappa_{E} f\right), \delta_{x} \otimes \phi\right\rangle=\left\langle\kappa_{E} f(x), \phi\right\rangle=\langle\phi, f(x)\rangle .
$$

Furthermore, $\left\|\Gamma\left(\kappa_{E} f\right)\right\|=\operatorname{Lip}\left(\kappa_{E} f\right)=\operatorname{Lip}(f)$. Since $\varepsilon \leq \pi$ on $\mathcal{F}(X) \otimes E^{*}$, we wish to study for which Lipschitz operators $f$ in $\operatorname{Lip}_{0}(X, E)$ the linear functional 
$\Gamma\left(\kappa_{E} f\right)$ is continuous even with respect to $\varepsilon$. Towards this end, we will need the class $\operatorname{Lip}_{0 G I}(X, E)$ of Lipschitz G-integral operators from $X$ to $E$, presented in the introduction.

In light of the next result, we first obtain that $\left(\operatorname{Lip}_{0 G I}(X, E), \operatorname{Lip}_{G I}\right)$ is a Banach space and a Banach ideal of Lipschitz operators.

Proposition 2.1. $\operatorname{Lip}_{0 G I}(X, E)$ is a linear subspace of $\operatorname{Lip}_{0}(X, E)$ satisfying the following properties:

(i) $\operatorname{Lip}(f) \leq \operatorname{Lip}_{G I}(f)$ for all $f \in \operatorname{Lip}_{0 G I}(X, E)$.

(ii) $\operatorname{Lip}_{G I}$ is a Banach space norm on $\operatorname{Lip}_{0 G I}(X, E)$.

(iii) If $g \in X^{\#}$ and $e \in E$, then $g \cdot e \in \operatorname{Lip}_{0 G I}(X, E)$ and $\operatorname{Lip}_{G I}(g \cdot e)=$ $\operatorname{Lip}(g)\|e\|$.

(iv) Let $Y$ be a pointed metric space and $F$ a Banach space. If $h \in \operatorname{Lip}_{0}(Y, X)$, $f \in \operatorname{Lip}_{0 G I}(X, E)$ and $T \in \mathcal{L}(E, F)$, then $T f h \in \operatorname{Lip}_{0 G I}(Y, F)$ and $\operatorname{Lip}_{G I}(T f h) \leq\|T\| \operatorname{Lip}_{G I}(f) \operatorname{Lip}(h)$.

Proof. (i) Let $f \in \operatorname{Lip}_{0 G I}(X, E)$ and let $(A, b, \mu)$ be a Lipschitz G-integral factorization for $f$. Clearly, $\kappa_{E} f \in \operatorname{Lip}_{0}\left(X, E^{* *}\right)$ and $\operatorname{Lip}\left(\kappa_{E} f\right)=\operatorname{Lip}(f)$. Moreover, $\operatorname{Lip}\left(\kappa_{E} f\right) \leq\|A\| \operatorname{Lip}(b) \mu(\Omega)$ and passing to the infimum yields $\operatorname{Lip}(f) \leq \operatorname{Lip}_{G I}(f)$.

(ii) We first show that $\operatorname{Lip}_{0 G I}(X, E)$ is a linear subspace of $\operatorname{Lip}_{0}(X, E)$. For any $\lambda \in \mathbb{K}$, the triple $(\lambda A, b, \mu)$ is a Lipschitz G-integral factorization of $\lambda f$. Then $\lambda f \in \operatorname{Lip}_{0 G I}(X, E)$ and

$$
\operatorname{Lip}_{G I}(\lambda f) \leq\|\lambda A\| \operatorname{Lip}(b) \mu(\Omega)=|\lambda|\|A\| \operatorname{Lip}(b) \mu(\Omega) .
$$

It follows that $\operatorname{Lip}_{G I}(\lambda f)=0=|\lambda| \operatorname{Lip}_{G I}(f)$ if $\lambda=0$, and that $\operatorname{Lip}_{G I}(\lambda f) \leq$ $|\lambda| \operatorname{Lip}_{G I}(f)$ if $\lambda \neq 0$. Then, for $\lambda \neq 0$, we have $\operatorname{Lip}_{G I}(f)=\operatorname{Lip}_{G I}\left(\lambda^{-1} \lambda f\right) \leq$ $|\lambda|^{-1} \operatorname{Lip}_{G I}(\lambda f)$, hence $|\lambda| \operatorname{Lip}_{G I}(f) \leq \operatorname{Lip}_{G I}(\lambda f)$ and so $\operatorname{Lip}_{G I}(\lambda f)=|\lambda| \operatorname{Lip}_{G I}(f)$.

Let $f_{1}, f_{2} \in \operatorname{Lip}_{0 G I}(X, E)$ and $\varepsilon>0$. For $i=1,2$ we can find a probability space $\left(\Omega_{i}, \Sigma_{i}, \mu_{i}\right)$, the formal inclusion operator $I_{\infty, 1, i}: L_{\infty}\left(\mu_{i}\right) \rightarrow L_{1}\left(\mu_{i}\right)$, a Lipschitz operator $b_{i} \in \operatorname{Lip}_{0}\left(X, L_{\infty}\left(\mu_{i}\right)\right)$ with $\operatorname{Lip}\left(b_{i}\right)=1 / 2$ and a linear operator $A_{i} \in$ $\mathcal{L}\left(L_{1}\left(\mu_{i}\right), E^{* *}\right)$ such that $\kappa_{E} f_{i}$ factors as

$$
\kappa_{E} f_{i}=A_{i} I_{\infty, 1, i} b_{i}: X \stackrel{b_{i}}{\rightarrow} L_{\infty}\left(\mu_{i}\right) \stackrel{I_{\infty, 1, i}}{\rightarrow} L_{1}\left(\mu_{i}\right) \stackrel{A_{i}}{\rightarrow} E^{* *}
$$

satisfying $\left\|A_{i}\right\|<\operatorname{Lip}_{G I}\left(f_{i}\right)+\varepsilon / 2$. We may assume also that $\Omega_{1} \cap \Omega_{2}=\emptyset$.

Take $\Omega:=\Omega_{1} \cup \Omega_{2}$ and $\Sigma:=\left\{S \subset \Omega: S \cap \Omega_{i} \in \Sigma_{i}, i=1,2\right\}$. Define the probability measure $\mu$ on $\Sigma$ by

$$
\mu(S)=\frac{\left\|A_{1}\right\| \mu_{1}\left(S \cap \Omega_{1}\right)+\left\|A_{2}\right\| \mu_{2}\left(S \cap \Omega_{2}\right)}{\left\|A_{1}\right\|+\left\|A_{2}\right\|} .
$$

Define $A: L_{1}(\mu) \rightarrow E^{* *}$ and $b: X \rightarrow L_{\infty}(\mu)$ by

$$
\begin{aligned}
A(s) & =A_{1}\left(\left.s\right|_{\Omega_{1}}\right)+A_{2}\left(\left.s\right|_{\Omega_{2}}\right), \\
b(x) & =b_{1}(x) \cdot \chi_{\Omega_{1}}+b_{2}(x) \cdot \chi_{\Omega_{2}},
\end{aligned}
$$

where $\chi_{\Omega_{i}}$ is the characteristic function of the subset $\Omega_{i} \subset \Omega$ for $i=1,2$. Clearly, $A$ is linear and

$$
\|A(s)\| \leq\left\|A_{1}\right\|\left\|\left.s\right|_{\Omega_{1}}\right\|_{L_{1}\left(\mu_{1}\right)}+\left\|A_{2}\right\|\left\|\left.s\right|_{\Omega_{2}}\right\|_{L_{1}\left(\mu_{2}\right)}=\left(\left\|A_{1}\right\|+\left\|A_{2}\right\|\right)\|s\|_{L_{1}(\mu)}
$$


for all $s \in L_{1}(\mu)$. Hence $A \in \mathcal{L}\left(L_{1}(\mu), E\right)$ and $\|A\| \leq\left\|A_{1}\right\|+\left\|A_{2}\right\|$. Moreover, $b \in \operatorname{Lip}_{0}\left(X, L_{\infty}(\mu)\right)$ with $\operatorname{Lip}(b) \leq 1$ because $b(0)=0$ and

$$
\begin{aligned}
\|b(x)-b(y)\|_{L_{\infty}(\mu)} & \leq\left\|\left(b_{1}(x)-b_{1}(y)\right) \cdot \chi_{\Omega_{1}}\right\|_{L_{\infty}(\mu)}+\left\|\left(b_{2}(x)-b_{2}(y)\right) \cdot \chi_{\Omega_{2}}\right\|_{L_{\infty}(\mu)} \\
& =\left\|b_{1}(x)-b_{1}(y)\right\|_{L_{\infty}\left(\mu_{1}\right)}+\left\|b_{2}(x)-b_{2}(y)\right\|_{L_{\infty}\left(\mu_{2}\right)} \\
& \leq d(x, y)\left(\operatorname{Lip}\left(b_{1}\right)+\operatorname{Lip}\left(b_{2}\right)\right) \\
& =d(x, y)
\end{aligned}
$$

for all $x, y \in X$. For each $x \in X$, we have

$$
\begin{aligned}
A I_{\infty, 1} b(x) & =A I_{\infty, 1}\left(b_{1}(x) \cdot \chi_{\Omega_{1}}+b_{2}(x) \cdot \chi_{\Omega_{2}}\right) \\
& =A\left(I_{\infty, 1}\left(b_{1}(x) \cdot \chi_{\Omega_{1}}\right)+I_{\infty, 1}\left(b_{2}(x) \cdot \chi_{\Omega_{2}}\right)\right) \\
& =\sum_{i=1}^{2} A_{i}\left(\left.\left(I_{\infty, 1}\left(b_{1}(x) \cdot \chi_{\Omega_{1}}\right)+I_{\infty, 1}\left(b_{2}(x) \cdot \chi_{\Omega_{2}}\right)\right)\right|_{\Omega_{i}}\right) \\
& =A_{1} I_{\infty, 1,1} b_{1}(x)+A_{2} I_{\infty, 1,2} b_{2}(x) \\
& =\kappa_{E} f_{1}(x)+\kappa_{E} f_{2}(x) \\
& =\kappa_{E}\left(f_{1}+f_{2}\right)(x),
\end{aligned}
$$

and thus $\kappa_{E}\left(f_{1}+f_{2}\right)=A I_{\infty, 1} b$. Hence $f_{1}+f_{2} \in \operatorname{Lip}_{0 G I}(X, E)$ and

$$
\operatorname{Lip}_{G I}\left(f_{1}+f_{2}\right) \leq\|A\| \operatorname{Lip}(b) \mu(\Omega) \leq\left\|A_{1}\right\|+\left\|A_{2}\right\| \leq \operatorname{Lip}_{G I}\left(f_{1}\right)+\operatorname{Lip}_{G I}\left(f_{2}\right)+\varepsilon
$$

Since $\varepsilon$ was arbitrary, it follows that $\operatorname{Lip}_{G I}\left(f_{1}+f_{2}\right) \leq \operatorname{Lip}_{G I}\left(f_{1}\right)+\operatorname{Lip}_{G I}\left(f_{2}\right)$.

We have proved above that $\operatorname{Lip}_{G I}$ is a norm on $\operatorname{Lip}_{0 G I}(X, E)$. To show that this norm is complete, it is enough to check that if $\left\{f_{n}\right\}_{n \in \mathbb{N}}$ is a sequence in $\operatorname{Lip}_{0 G I}(X, E)$ such that $\sum_{n=1}^{\infty} \operatorname{Lip}_{G I}\left(f_{n}\right)<\infty$, then the series $\sum f_{n}$ is convergent in $\left(\operatorname{Lip}_{0 G I}(X, E), \operatorname{Lip}_{G I}\right)$. To prove this, we follow the proof of [6, Theorem 5.2 (a)].

By (i), $\sum_{n=1}^{\infty} \operatorname{Lip}\left(f_{n}\right)<\infty$ too, and so $f=\sum_{n=1}^{\infty} f_{n}$ exists in $\operatorname{Lip}_{0}(X, E)$. We claim that $f \in \operatorname{Lip}_{0 G I}(X, E)$ and $\operatorname{Lip}_{G I}(f) \leq \sum_{n=1}^{\infty} \operatorname{Lip}_{G I}\left(f_{n}\right)$. Indeed, let $\varepsilon>0$. For each $n \in \mathbb{N}$ we can find a probability space $\left(\Omega_{n}, \Sigma_{n}, \mu_{n}\right)$, the formal inclusion operator $I_{\infty, 1, n}: L_{\infty}\left(\mu_{n}\right) \rightarrow L_{1}\left(\mu_{n}\right)$, a Lipschitz operator $b_{n} \in \operatorname{Lip}_{0}\left(X, L_{\infty}\left(\mu_{n}\right)\right)$ with $\operatorname{Lip}\left(b_{n}\right)=1 / 2^{n}$ and a linear operator $A_{n} \in \mathcal{L}\left(L_{1}\left(\mu_{n}\right), E^{* *}\right)$ such that $\kappa_{E} f_{n}$ factors as

$$
\kappa_{E} f_{n}=A_{n} I_{\infty, 1, n} b_{n}: X \stackrel{b_{n}}{\rightarrow} L_{\infty}\left(\mu_{n}\right) \stackrel{I_{\infty, 1, n}}{\rightarrow} L_{1}\left(\mu_{n}\right) \stackrel{A_{n}}{\rightarrow} E^{* *}
$$

with $\left\|A_{n}\right\|<\operatorname{Lip}_{G I}\left(f_{n}\right)+\varepsilon / 2^{n}$. Let $(\Omega, \Sigma)$ be the direct sum measurable space of the $\left(\Omega_{n}, \Sigma_{n}\right)$, that is, $\Omega:=\cup_{n \in \mathbb{N}} \Omega_{n}$ and $\Sigma:=\left\{S \subset \Omega: S \cap \Omega_{n} \in \Sigma_{n}, \forall n \in \mathbb{N}\right\}$ where the $\Omega_{n}$ 's are pairwise disjoint. Define a probability measure $\mu$ on $\Sigma$ by

$$
\mu\left(S_{m}\right)=\mu_{m}\left(S_{m}\right) \frac{\left\|A_{m}\right\|}{\sum_{n=1}^{\infty}\left\|A_{n}\right\|} \quad\left(m \in \mathbb{N}, S_{m} \in \Sigma_{m}\right) .
$$


Define $A: L_{1}(\mu) \rightarrow E^{* *}$ and $b: X \rightarrow L_{\infty}(\mu)$ by

$$
\begin{aligned}
& A(s)=\sum_{n=1}^{\infty} A_{n}\left(\left.s\right|_{\Omega_{n}}\right), \\
& b(x)=\sum_{n=1}^{\infty} b_{n}(x) \cdot \chi_{\Omega_{n}} .
\end{aligned}
$$

Clearly, $A$ is linear and, for any $s \in L_{1}(\mu)$, we have

$$
\|A(s)\| \leq \sum_{n=1}^{\infty}\left\|A_{n}\right\|\left\|\left.s\right|_{\Omega_{n}}\right\|_{L_{1}\left(\mu_{n}\right)} \leq\|s\|_{L_{1}(\mu)} \sum_{n=1}^{\infty}\left\|A_{n}\right\| .
$$

Hence $A \in \mathcal{L}\left(L_{1}(\mu), E\right)$ and $\|A\| \leq \sum_{n=1}^{\infty}\left\|A_{n}\right\| \leq \sum_{n=1}^{\infty} \operatorname{Lip}_{G I}\left(f_{n}\right)+\varepsilon$. Moreover, $b \in \operatorname{Lip}_{0}\left(X, L_{\infty}(\mu)\right)$ with $\operatorname{Lip}(b) \leq 1$ because $b(0)=0$ and

$$
\begin{aligned}
\|b(x)-b(y)\|_{L_{\infty}(\mu)} & \leq \sum_{n=1}^{\infty}\left\|\left(b_{n}(x)-b_{n}(y)\right) \cdot \chi_{\Omega_{n}}\right\|_{L_{\infty}(\mu)} \\
& =\sum_{n=1}^{\infty}\left\|b_{n}(x)-b_{n}(y)\right\|_{L_{\infty}\left(\mu_{n}\right)} \\
& \leq d(x, y) \sum_{n=1}^{\infty} \operatorname{Lip}\left(b_{n}\right)=d(x, y)
\end{aligned}
$$

for all $x, y \in X$. For each $x \in X$, we have

$$
\begin{aligned}
A I_{\infty, 1} b(x) & =A I_{\infty, 1}\left(\sum_{n=1}^{\infty} b_{n}(x) \cdot \chi_{\Omega_{n}}\right) \\
& =A\left(\sum_{n=1}^{\infty} I_{\infty, 1}\left(b_{n}(x) \cdot \chi_{\Omega_{n}}\right)\right) \\
& =\sum_{m=1}^{\infty} A_{m}\left(\left.\left(\sum_{n=1}^{\infty} I_{\infty, 1}\left(b_{n}(x) \cdot \chi_{\Omega_{n}}\right)\right)\right|_{\Omega_{m}}\right) \\
& =\sum_{m=1}^{\infty} A_{m} I_{\infty, 1, m} b_{m}(x) \\
& =\sum_{m=1}^{\infty} \kappa_{E} f_{m}(x)=\kappa_{E} f(x),
\end{aligned}
$$

and thus $\kappa_{E} f=A I_{\infty, 1} b$. Hence $f \in \operatorname{Lip}_{0 G I}(X, E)$ and

$$
\operatorname{Lip}_{G I}(f) \leq\|A\| \operatorname{Lip}(b) \mu(\Omega) \leq \sum_{n=1}^{\infty} \operatorname{Lip}_{G I}\left(f_{n}\right)+\varepsilon .
$$

By the arbitrariness of $\varepsilon$, we infer that $\operatorname{Lip}_{G I}(f) \leq \sum_{n=1}^{\infty} \operatorname{Lip}_{G I}\left(f_{n}\right)$ and this proves our claim.

We now will show that $f$ is the $\operatorname{Lip}_{G I}$-limit of the sequence $\left\{\sum_{k=1}^{n} f_{k}\right\}_{n \in \mathbb{N}}$. For each $n \in \mathbb{N}$, define $t_{n}: L_{1}(\mu) \rightarrow E^{* *}$ by $t_{n}(s)=\sum_{k=n+1}^{\infty} A_{k}\left(\left.s\right|_{\Omega_{k}}\right)$. Clearly, 
$t_{n} \in \mathcal{L}\left(L_{1}(\mu), E^{* *}\right)$ with $\left\|t_{n}\right\| \leq \sum_{k=n+1}^{\infty}\left\|A_{k}\right\|$ and so $\lim _{n \rightarrow \infty}\left\|t_{n}\right\|=0$. It is easy to see that $f-\sum_{k=1}^{n} f_{k}=t_{n} I_{\infty, 1} b$. Then $\operatorname{Lip}_{G I}\left(f-\sum_{k=1}^{n} f_{k}\right) \leq\left\|t_{n}\right\| \operatorname{Lip}(b)$ and therefore $\lim _{n \rightarrow \infty} \operatorname{Lip}_{G I}\left(f-\sum_{k=1}^{n} f_{k}\right)=0$ as desired.

(iii) Fix a point $x_{0} \in X$ and take $\Omega=\left\{x_{0}\right\}, \Sigma=\{\Omega, \emptyset\}$ and $\mu: \Sigma \rightarrow \mathbb{R}$ defined by $\mu(\Omega)=1$ and $\mu(\emptyset)=0$. Then $(\Omega, \Sigma, \mu)$ is a probability space. Clearly, $L_{\infty}(\mu)$ and $L_{1}(\mu)$ contain only constant functions.

Let $g \in X^{\#}$ and $e \in E$. Define $A \in \mathcal{L}\left(L_{1}(\mu), E^{* *}\right)$ and $b \in \operatorname{Lip}_{0}\left(X, L_{\infty}(\mu)\right)$ by $A(t \mathbf{1})=t \kappa_{E}(e)$ for all $t \in \mathbb{K}$ and $b(x)=g(x) \mathbf{1}$ for all $x \in X$, where $\mathbf{1}$ is the function constantly equal to 1 on $\Omega$. It is clear that

$$
\left(\kappa_{E}(g \cdot e)\right)(x)=g(x) \kappa_{E}(e)=g(x) A(\mathbf{1})=A(g(x) \mathbf{1})=A I_{\infty, 1}(g(x) \mathbf{1})=A I_{\infty, 1} b(x)
$$

for all $x \in X$. Then $g \cdot e \in \operatorname{Lip}_{0 G I}(X, E)$ and $\operatorname{Lip}_{G I}(g \cdot e) \leq\|A\| \operatorname{Lip}(b)=$ $\|e\| \operatorname{Lip}(g)$. The converse inequality follows from (i) and Proposition 1.1 (ii).

(iv) Since $f \in \operatorname{Lip}_{0 G I}(X, E)$, consider a typical Lipschitz G-integral factorization

$$
\kappa_{E} f=A I_{\infty, 1} b: X \stackrel{b}{\rightarrow} L_{\infty}(\mu) \stackrel{I_{\infty, 1}}{\rightarrow} L_{1}(\mu) \stackrel{A}{\rightarrow} E^{* *} .
$$

Since $\kappa_{F} T=T^{* *} \kappa_{E}$, putting

$$
B=T^{* *} A \in \mathcal{L}\left(L_{1}(\mu), F^{* *}\right), \quad c=b h \in \operatorname{Lip}_{0}\left(Y, L_{\infty}(\mu)\right),
$$

we obtain

$$
\kappa_{F} T f h=B I_{\infty, 1} c: Y \stackrel{c}{\rightarrow} L_{\infty}(\mu) \stackrel{I_{\infty, 1}}{\rightarrow} L_{1}(\mu) \stackrel{B}{\rightarrow} F^{* *} .
$$

Hence $T f h \in \operatorname{Lip}_{0 G I}(Y, F)$ and the inequality $\operatorname{Lip}_{G I}(T f h) \leq\|T\| \operatorname{Lip}_{G I}(f) \operatorname{Lip}(h)$ follows readily from

$$
\operatorname{Lip}_{G I}(T f h) \leq\|B\| \operatorname{Lip}(c) \mu(\Omega) \leq\|T\|\|A\| \operatorname{Lip}(b) \operatorname{Lip}(h) \mu(\Omega) .
$$

We now may answer for the question which opened this section.

Theorem 2.2. A Lipschitz operator $f \in \operatorname{Lip}_{0}(X, E)$ is a Lipschitz G-integral operator if and only if its associate linear functional $\Gamma\left(\kappa_{E} f\right)$ on $\mathcal{F}(X) \otimes E^{*}$ is continuous on $\mathcal{F}(X) \widehat{\otimes}_{\varepsilon} E^{*}$. Furthermore, $\operatorname{Lip}_{G I}(f)=\left\|\Gamma\left(\kappa_{E} f\right)\right\|$.

Proof. Assume that $\Gamma\left(\kappa_{E} f\right)$ is a bounded linear functional on $\mathcal{F}(X) \widehat{\otimes}_{\varepsilon} E^{*}$. By Theorem 1.3, there exist a finite measure space $(\Omega, \Sigma, \mu)$ and operators $B \in$ $\mathcal{L}\left(E^{*}, L_{\infty}(\mu)\right)$ and $b \in \operatorname{Lip}_{0}\left(X, L_{\infty}(\mu)\right)$ such that

$$
\left\langle\Gamma\left(\kappa_{E} f\right), \delta_{x} \otimes \phi\right\rangle=\int_{\Omega} b(x) B(\phi) d \mu
$$

for all $x \in X$ and $\phi \in E^{*}$, with $\left\|\Gamma\left(\kappa_{E} f\right)\right\|=\|B\| \operatorname{Lip}(b) \mu(\Omega)$. Define

$$
A=B^{*} \Phi^{*} \kappa_{L_{1}(\mu)}: L_{1}(\mu) \stackrel{\kappa_{L_{1}(\mu)}}{\rightarrow} L_{1}(\mu)^{* *} \stackrel{\Phi^{*}}{\rightarrow} L_{\infty}(\mu)^{*} \stackrel{B^{*}}{\rightarrow} E^{* *} .
$$

It is clear that $A \in \mathcal{L}\left(L_{1}(\mu), E^{* *}\right)$ and, for any $x \in X$ and $\phi \in E^{*}$, we have

$$
\begin{gathered}
\left\langle\kappa_{E} f(x), \phi\right\rangle=\left\langle\Gamma\left(\kappa_{E} f\right), \delta_{x} \otimes \phi\right\rangle=\int_{\Omega} b(x) B(\phi) d \mu=\int_{\Omega} I_{\infty, 1} B(\phi) I_{\infty, 1} b(x) d \mu \\
=\left\langle\Phi(B(\phi)), I_{\infty, 1} b(x)\right\rangle=\left\langle\kappa_{L_{1}(\mu)}\left(I_{\infty, 1} b(x)\right), \Phi(B(\phi))\right\rangle \\
=\left\langle B^{*} \Phi^{*} \kappa_{L_{1}(\mu)} I_{\infty, 1} b(x), \phi\right\rangle=\left\langle A I_{\infty, 1} b(x), \phi\right\rangle
\end{gathered}
$$


and thus

$$
\kappa_{E} f=A I_{\infty, 1} b: X \stackrel{b}{\rightarrow} L_{\infty}(\mu) \stackrel{I_{\infty, 1}}{\rightarrow} L_{1}(\mu) \stackrel{A}{\rightarrow} E^{* *} .
$$

Moreover,

$$
\begin{aligned}
\|A\| & =\sup _{s \in B_{L_{1}(\mu)}}\left\|B^{*} \Phi^{*} \kappa_{L_{1}(\mu)}(s)\right\| \\
& =\sup _{s \in B_{L_{1}(\mu)}} \sup _{\phi \in B_{E^{*}}}\left|\left\langle B^{*} \Phi^{*} \kappa_{L_{1}(\mu)}(s), \phi\right\rangle\right| \\
& =\sup _{\phi \in B_{E^{*}}} \sup _{s \in B_{L_{1}(\mu)}}|\langle\Phi(B(\phi)), s\rangle| \\
& =\sup _{\phi \in B_{E^{*}}}\|\Phi(B(\phi))\| \\
& =\sup _{\phi \in B_{E^{*}}}\|B(\phi)\|=\|B\|,
\end{aligned}
$$

and therefore $\operatorname{Lip}_{G I}(f) \leq\|A\| \operatorname{Lip}(b) \mu(\Omega)=\left\|\Gamma\left(\kappa_{E} f\right)\right\|$.

Conversely, assume that $f$ has a Lipschitz G-integral factorization of the form

$$
\kappa_{E} f=A I_{\infty, 1} b: X \stackrel{b}{\rightarrow} L_{\infty}(\mu) \stackrel{I_{\infty, 1}}{\rightarrow} L_{1}(\mu) \stackrel{A}{\rightarrow} E^{* *} .
$$

Take

$$
B=\Phi^{-1} A^{*} \kappa_{E^{*}}: E^{*} \stackrel{\kappa_{E^{*}}}{\rightarrow} E^{* * *} \stackrel{A^{*}}{\rightarrow} L_{1}(\mu)^{*} \stackrel{\Phi^{-1}}{\rightarrow} L_{\infty}(\mu) .
$$

Clearly, $B \in \mathcal{L}\left(E^{*}, L_{\infty}(\mu)\right)$ and $\|B\| \leq\|A\|$. For $x \in X$ and $\phi \in E^{*}$, we have

$$
\begin{gathered}
\left\langle\Gamma\left(\kappa_{E} f\right), \delta_{x} \otimes \phi\right\rangle=\left\langle\kappa_{E} f(x), \phi\right\rangle=\left\langle\kappa_{E^{*}}(\phi), \kappa_{E} f(x)\right\rangle \\
=\left\langle\kappa_{E^{*}}(\phi), A I_{\infty, 1} b(x)\right\rangle=\left\langle A^{*} \kappa_{E^{*}}(\phi), I_{\infty, 1} b(x)\right\rangle=\left\langle\Phi(B(\phi)), I_{\infty, 1} b(x)\right\rangle \\
=\int_{\Omega} I_{\infty, 1} b(x) I_{\infty, 1} B(\phi) d \mu=\int_{\Omega} b(x) B(\phi) d \mu .
\end{gathered}
$$

Hence $\Gamma\left(\kappa_{E} f\right)$ is continuous on $\mathcal{F}(X) \widehat{\otimes}_{\varepsilon} E^{*}$ and $\left\|\Gamma\left(\kappa_{E} f\right)\right\| \leq\|B\| \operatorname{Lip}(b) \mu(\Omega) \leq$ $\|A\| \operatorname{Lip}(b) \mu(\Omega)$ by Theorem 1.3. The factorization $\kappa_{E} f=A I_{\infty, 1} b$ was arbitrary, so $\left\|\Gamma\left(\kappa_{E} f\right)\right\| \leq \operatorname{Lip}_{G I}(f)$.

The space $L_{\infty}(\mu)$ in Definition 1 can be replaced by the space $C_{w^{*}}\left(B_{X^{\#}}\right)$ of all weak* continuous functions from $B_{X \#}$ into $\mathbb{K}$. Let us recall that a Banach space $F$ is injective if, whenever $E_{0}$ is a subspace of a Banach space $E$, any $T \in \mathcal{L}\left(E_{0}, F\right)$ has an extension $\widetilde{T} \in \mathcal{L}(E, F)$ with $\|T\|=\|\widetilde{T}\|$. For any finite measure, $L_{\infty}(\mu)$ is an injective Banach space by [6, Theorem 4.14].

Theorem 2.3. A Lipschitz operator $f \in \operatorname{Lip}_{0}(X, E)$ is a Lipschitz G-integral operator if and only if there exist a positive regular Borel measure $\nu$ on the Borel $\sigma$-algebra of $B_{X^{\#}}$ with the weak ${ }^{*}$ topology and an operator $\widetilde{A} \in \mathcal{L}\left(L_{1}(\nu), E^{* *}\right)$ such that the following diagram commutes:

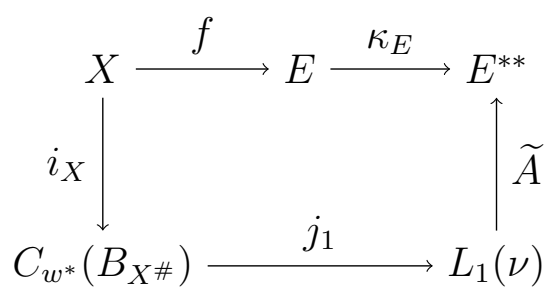


where $j_{1}$ is the canonical map and $i_{X}$ is the natural isometric embedding defined by $i_{X}(x)=\left.\delta_{x}\right|_{B_{X \#}}$. In this case, $\operatorname{Lip}_{G I}(f)=\inf \|\widetilde{A}\| \nu\left(B_{X \#}\right)$, where the infimum is taken over all such possible $\nu$ 's and $\widetilde{A}$ 's, and this infimum is attained.

Proof. Let $f \in \operatorname{Lip}_{0}(X, E)$ and assume that there exist a positive regular Borel measure $\nu$ on the Borel $\sigma$-algebra of $B_{X \#}$ with the weak* topology and an operator $\widetilde{A} \in \mathcal{L}\left(L_{1}(\nu), E^{* *}\right)$ such that

$$
\kappa_{E} f=\widetilde{A} j_{1} i_{X}: X \stackrel{i_{X}}{\rightarrow} C_{w^{*}}\left(B_{X^{\#}}\right) \stackrel{j_{1}}{\rightarrow} L_{1}(\nu) \stackrel{\widetilde{A}}{\rightarrow} E^{* *} .
$$

Take the canonical map $j_{\infty}: C_{w^{*}}\left(B_{X^{\#}}\right) \rightarrow L_{\infty}(\nu)$ and factor

$$
j_{1}=I_{\infty, 1} j_{\infty}: C_{w^{*}}\left(B_{X^{\#}}\right) \stackrel{j_{\infty}}{\rightarrow} L_{\infty}(\nu) \stackrel{I_{\infty}, 1}{\rightarrow} L_{1}(\nu) .
$$

Denoting $b=j_{\infty} i_{X} \in \operatorname{Lip}_{0}\left(X, L_{\infty}(\nu)\right)$, we have

$$
\kappa_{E} f=\widetilde{A} I_{\infty, 1} b: X \stackrel{b}{\rightarrow} L_{\infty}(\nu) \stackrel{I_{\infty, 1}}{\rightarrow} L_{1}(\nu) \stackrel{\widetilde{A}}{\rightarrow} E^{* *} .
$$

Then $f \in \operatorname{Lip}_{0 G I}(X, E)$ and $\operatorname{Lip}_{G I}(f) \leq\left\|\widetilde{A}|| \operatorname{Lip}(b) \nu\left(B_{X^{\#}}\right) \leq\right\| \widetilde{A} \| \nu\left(B_{X^{\#}}\right)$.

Conversely, suppose that $f \in \operatorname{Lip}_{0 G I}(X, E)$. Then there are a finite measure space $(\Omega, \Sigma, \mu)$ and operators $A \in \mathcal{L}\left(L_{1}(\mu), E^{* *}\right)$ and $b \in \operatorname{Lip}_{0}\left(X, L_{\infty}(\mu)\right)$ such that

$$
\kappa_{E} f=A I_{\infty, 1} b: X \stackrel{b}{\rightarrow} L_{\infty}(\mu) \stackrel{I_{\infty, 1}}{\rightarrow} L_{1}(\mu) \stackrel{A}{\rightarrow} E^{* *} .
$$

Let $\mathcal{F}(X)$ be the Lipschitz-free space over $X$. By Theorem 1.2, there exists $T_{b} \in \mathcal{L}\left(\mathcal{F}(X), L_{\infty}(\mu)\right)$ such that $T_{b} \delta_{X}=b$ and $\left\|T_{b}\right\|=\operatorname{Lip}(b)$. Moreover, $\mathcal{F}(X)$ is the space of all linear functionals $\gamma$ on $X^{\#}$ such that $\left.\gamma\right|_{B_{X} \#}$ is weak* continuous. Denote by $R$ the linear map $\left.\gamma \mapsto \gamma\right|_{B_{X} \#}$ from $\mathcal{F}(X)$ into $C_{w^{*}}\left(B_{X^{\#}}\right)$. Since $L_{\infty}(\mu)$ is injective, there is an operator $\widetilde{T}_{b} \in \mathcal{L}\left(C_{w^{*}}\left(B_{X^{\#}}\right), L_{\infty}(\mu)\right)$ such that $\widetilde{T}_{b} R=T_{b}$ and $\left\|T_{b}\right\|=\left\|\widetilde{T}_{b}\right\|$. It is known (see $[6,2.9(\mathrm{~d})$ and 2.4]) that $I_{\infty, 1}: L_{\infty}(\mu) \rightarrow L_{1}(\mu)$ is 1 -summing with 1 -summing norm $\pi_{1}\left(I_{\infty, 1}\right)=\mu(\Omega)$, and so is $I_{\infty, 1} \widetilde{T}_{b} \in \mathcal{L}\left(C_{w^{*}}\left(B_{X \#}\right), L_{1}(\mu)\right)$ with $\pi_{1}\left(I_{\infty, 1} \widetilde{T}_{b}\right) \leq \pi_{1}\left(I_{\infty, 1}\right)\left\|\widetilde{T}_{b}\right\|$. Then, applying [6, Corollary 2.15] to $I_{\infty, 1} \widetilde{T}_{b}$, we may obtain a positive regular Borel measure $\nu$ on the Borel $\sigma$-algebra of $B_{X^{\#}}$ with the weak* topology and an operator $C \in \mathcal{L}\left(L_{1}(\nu), L_{1}(\mu)\right)$ such that

$$
I_{\infty, 1} \widetilde{T}_{b}=C j_{1}: C_{w^{*}}\left(B_{X \#}\right) \stackrel{j_{1}}{\rightarrow} L_{1}(\nu) \stackrel{C}{\rightarrow} L_{1}(\mu)
$$

and $\pi_{1}\left(I_{\infty, 1} \widetilde{T}_{b}\right)=\|C\| \nu\left(B_{X \#}\right)$. Put $\widetilde{A}=A C \in \mathcal{L}\left(L_{1}(\nu), E^{* *}\right)$. An easy verification yields

$$
\kappa_{E} f=A I_{\infty, 1} b=A I_{\infty, 1} T_{b} \delta_{X}=A I_{\infty, 1} \widetilde{T}_{b} R \delta_{X}=A C j_{1} R \delta_{X}=\widetilde{A} j_{1} i_{X}
$$

and so we have

$$
\kappa_{E} f=\widetilde{A} j_{1} i_{X}: X \stackrel{i_{X}}{\rightarrow} C_{w^{*}}\left(B_{X^{\#}}\right) \stackrel{j_{1}}{\rightarrow} L_{1}(\nu) \stackrel{\widetilde{A}}{\rightarrow} E^{* *} .
$$

This is a factorization of $\kappa_{E} f$ in the desired form. It follows that

$$
\begin{gathered}
\|\widetilde{A}\| \nu\left(B_{X^{\#}}\right) \leq\|A\|\|C\| \nu\left(B_{X^{\#}}\right)=\|A\| \pi_{1}\left(I_{\infty, 1} \widetilde{T}_{b}\right) \\
\leq\|A\|\left\|\widetilde{T}_{b}\right\| \pi_{1}\left(I_{\infty, 1}\right)=\|A\| \operatorname{Lip}(b) \mu(\Omega)
\end{gathered}
$$


Passing to the infimum we arrive at $\|\widetilde{A}\| \nu\left(B_{X^{\#}}\right) \leq \operatorname{Lip}_{G I}(f)$.

Let us recall that a linear operator $T: E \rightarrow F$ between Banach spaces is an integral operator if there are a finite measure space $(\Omega, \Sigma, \mu)$ and operators $A \in \mathcal{L}\left(L_{1}(\mu), F^{* *}\right)$ and $B \in \mathcal{L}\left(E, L_{\infty}(\mu)\right)$ giving rise to the commutative diagram

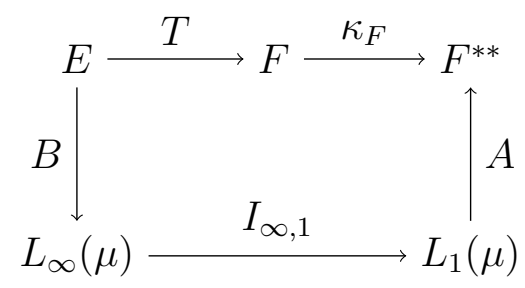

The integral norm of $T$ is $\|T\|_{I}=\inf \|A\|\|B\| \mu(\Omega)$, where the infimum is extended over all measures $\mu$ and operators $A$ and $B$ as above. We denote by $\mathcal{I}(E, F)$ the Banach space of all linear integral operators from $E$ to $F$ endowed with the integral norm. We refer to $[6,11]$ for a complete study of these operators.

We now obtain some nice characterizations of Lipschitz G-integral operators.

Proposition 2.4. Let $f \in \operatorname{Lip}_{0}(X, E)$. The following statements are equivalent:

(i) $f \in \operatorname{Lip}_{0 G I}(X, E)$.

(ii) $f^{t} \in \mathcal{I}\left(E^{*}, X^{\#}\right)$.

(iii) $T_{f} \in \mathcal{I}(\mathcal{F}(X), E)$.

Furthermore, if $f \in \operatorname{Lip}_{0 G I}(X, E)$, then $\operatorname{Lip}_{G I}(f)=\left\|f^{t}\right\|_{I}=\left\|T_{f}\right\|_{I}$.

Proof. (i) $\Rightarrow$ (ii): Assume that $f \in \operatorname{Lip}_{0 G I}(X, E)$ and select a typical factorization

$$
\kappa_{E} f=A I_{\infty, 1} b: X \stackrel{b}{\rightarrow} L_{\infty}(\mu) \stackrel{I_{\infty, 1}}{\rightarrow} L_{1}(\mu) \stackrel{A}{\rightarrow} E^{* *} .
$$

Then we have $f^{t} \kappa_{E}^{*} \kappa_{E^{*}}=b^{t} I_{\infty, 1}^{*} A^{*} \kappa_{E^{*}}$. Now notice that

$$
\kappa_{E}^{*} \kappa_{E^{*}}=I d_{E^{*}}: E^{*} \stackrel{\kappa_{E^{*}}}{\rightarrow} E^{* * *} \stackrel{\kappa_{E}^{*}}{\rightarrow} E^{*}
$$

and

$$
I_{\infty, 1}^{*}=\Phi^{*} \kappa_{L_{1}(\mu)} I_{\infty, 1} \Phi^{-1}: L_{1}(\mu)^{*} \stackrel{\Phi^{-1}}{\rightarrow} L_{\infty}(\mu) \stackrel{I_{\infty, 1}}{\rightarrow} L_{1}(\mu) \stackrel{\kappa_{L_{1}(\mu)}}{\rightarrow} L_{1}(\mu)^{* *} \stackrel{\Phi^{*}}{\rightarrow} L_{\infty}(\mu)^{*} .
$$

Indeed, for any $e \in E$ and $\phi \in E^{*}$, we have

$$
\left\langle\kappa_{E}^{*} \kappa_{E^{*}}(\phi), e\right\rangle=\left\langle\kappa_{E^{*}}(\phi), \kappa_{E}(e)\right\rangle=\left\langle\kappa_{E}(e), \phi\right\rangle=\langle\phi, e\rangle
$$

and, for any $t \in L_{\infty}(\mu)$ and $\varphi \in L_{1}(\mu)^{*}$,

$$
\begin{gathered}
\left\langle\Phi^{*} \kappa_{L_{1}(\mu)} I_{\infty, 1} \Phi^{-1}(\varphi), t\right\rangle=\left\langle\kappa_{L_{1}(\mu)} I_{\infty, 1} \Phi^{-1}(\varphi), \Phi(t)\right\rangle=\left\langle\Phi(t), I_{\infty, 1} \Phi^{-1}(\varphi)\right\rangle \\
=\int_{\Omega} I_{\infty, 1}(t) I_{\infty, 1} \Phi^{-1}(\varphi) d \mu=\left\langle\varphi, I_{\infty, 1}(t)\right\rangle=\left\langle I_{\infty, 1}^{*}(\varphi), t\right\rangle .
\end{gathered}
$$

Put $C=\kappa_{X} b^{t} \Phi^{*} \kappa_{L_{1}(\mu)} \in \mathcal{L}\left(L_{1}(\mu),\left(X^{\#}\right)^{* *}\right)$ and $D=\Phi^{-1} A^{*} \kappa_{E^{*}} \in \mathcal{L}\left(E^{*}, L_{\infty}(\mu)\right)$. Then we have the factorization

$$
\kappa_{X \#} f^{t}=C I_{\infty, 1} D: E^{*} \stackrel{D}{\rightarrow} L_{\infty}(\mu) \stackrel{I_{\infty, 1}}{\rightarrow} L_{1}(\mu) \stackrel{C}{\rightarrow}\left(X^{\#}\right)^{* *} .
$$

Then $f^{t} \in \mathcal{I}\left(E^{*}, X^{\#}\right)$ and

$$
\left\|f^{t}\right\|_{I} \leq\|C\|\|D\| \mu(\Omega) \leq\left\|b^{t}\right\|\left\|A^{*}\right\| \mu(\Omega)=\|A\| \operatorname{Lip}(b) \mu(\Omega) .
$$


The factorization $\kappa_{E} f=A I_{\infty, 1} b$ was arbitrary, so $\left\|f^{t}\right\|_{I} \leq \operatorname{Lip}_{G I}(f)$.

(ii) $\Rightarrow$ (i): Assume that $f^{t} \in \mathcal{I}\left(E^{*}, X^{\#}\right)$. Since $X^{\#}$ is a dual space, by [6, Corollary 5.4] there exist a finite measure space $(\Omega, \Sigma, \mu)$ and a pair of operators $A \in \mathcal{L}\left(L_{1}(\mu), X^{\#}\right)$ and $B \in \mathcal{L}\left(E^{*}, L_{\infty}(\mu)\right)$ such that $f^{t}=A I_{\infty, 1} B$. Let $\delta_{X}$ from $X$ to $\mathcal{F}(X)$ be the Dirac map. Then we have $\left(f^{t}\right)^{*} \delta_{X}=B^{*} I_{\infty, 1}^{*} A^{*} \delta_{X}$. Now notice that $\left(f^{t}\right)^{*} \delta_{X}=\kappa_{E} f$ since

$$
\begin{gathered}
\left\langle\left(f^{t}\right)^{*} \delta_{X}(x), \phi\right\rangle=\left\langle\left(f^{t}\right)^{*}\left(\delta_{x}\right), \phi\right\rangle=\left\langle\delta_{x}, f^{t}(\phi)\right\rangle \\
=\left\langle\delta_{x}, \phi \circ f\right\rangle=\langle\phi, f(x)\rangle=\left\langle\kappa_{E} f(x), \phi\right\rangle
\end{gathered}
$$

for every $\phi \in E^{*}$ and $x \in X$. By above-proved, $I_{\infty, 1}^{*}=\Phi^{*} \kappa_{L_{1}(\mu)} I_{\infty, 1} \Phi^{-1}$. Denote $C=B^{*} \Phi^{*} \kappa_{L_{1}(\mu)} \in \mathcal{L}\left(L_{1}(\mu), E^{* *}\right)$ and $b=\Phi^{-1} A^{*} \delta_{X} \in \operatorname{Lip}_{0}\left(X, L_{\infty}(\mu)\right)$. Then we have the factorization

$$
\kappa_{E} f=C I_{\infty, 1} b: X \stackrel{b}{\rightarrow} L_{\infty}(\mu) \stackrel{I_{\infty, 1}}{\rightarrow} L_{1}(\mu) \stackrel{C}{\rightarrow} E^{* *} .
$$

Hence $f \in \operatorname{Lip}_{0 G I}(X, E)$ with $\operatorname{Lip}_{G I}(f) \leq\|C\| \operatorname{Lip}(b) \mu(\Omega) \leq\left\|B^{*}\right\|\left\|A^{*}\right\| \mu(\Omega)=$ $\|A\|\|B\| \mu(\Omega)$, from which $\operatorname{Lip}_{G I}(f) \leq \inf \|A\|\|B\| \mu(\Omega)=\left\|f^{t}\right\|_{I}$.

(ii) $\Leftrightarrow$ (iii): Taking into account the ideal property of the space of integral linear operators between Banach spaces and the fact that such an operator is integral if and only if so is its adjoint operator, our equivalence follows immediately from the equality $f^{t}=Q_{X}^{-1}\left(T_{f}\right)^{*}$ given in Theorem 1.2. Moreover, $\left\|f^{t}\right\|_{I}=\left\|Q_{X}^{-1}\left(T_{f}\right)^{*}\right\|_{I}=$ $\left\|\left(T_{f}\right)^{*}\right\|_{I}=\left\|T_{f}\right\|_{I}$.

\section{Lipschitz Pietsch-INTEgRAL Operators}

If we wish to factor the Lipschitz operator $f \in \operatorname{Lip}_{0}(X, E)$ rather than $\kappa_{E} f$ in Definition 1, we obtain the following subclass of Lipschitz G-integral operators.

Definition 3. Let $X$ be a pointed metric space and let $E$ be a Banach space. A Lipschitz operator $f \in \operatorname{Lip}_{0}(X, E)$ is a Lipschitz Pietsch-integral (P-integral for short) operator if there exist a finite measure space $(\Omega, \Sigma, \mu)$, a bounded linear operator $A \in \mathcal{L}\left(L_{1}(\mu), E\right)$ and a Lipschitz operator $b \in \operatorname{Lip}_{0}\left(X, L_{\infty}(\mu)\right)$ such that the following diagram commutes:

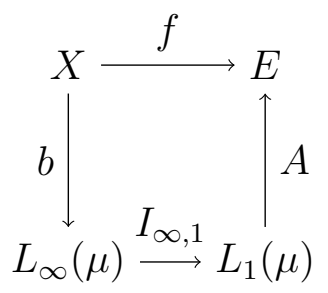

The triple $(A, b, \mu)$ is called a Lipschitz P-integral factorization of $f$. The Lipschitz P-integral norm of $f$ is defined to be $\operatorname{Lip}_{P I}(f)=\inf \|A\| \operatorname{Lip}(b) \mu(\Omega)$, where the infimum is extended over all Lipschitz P-integral factorizations of $f$. The set of all Lipschitz P-integral operators from $X$ to $E$ is denoted by $\operatorname{Lip}_{0 P I}(X, E)$.

Let us recall that a linear operator $T: E \rightarrow F$ between Banach spaces is a Pietsch integral (P-integral for short) operator if there are a finite measure space 
$(\Omega, \Sigma, \mu)$ and operators $A \in \mathcal{L}\left(L_{1}(\mu), F\right)$ and $B \in \mathcal{L}\left(E, L_{\infty}(\mu)\right)$ giving rise to the commutative diagram

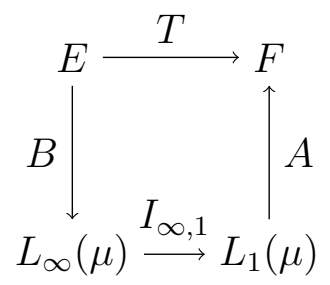

The P-integral norm of $T$ is $\|T\|_{P I}=\inf \|A\|\|B\| \mu(\Omega)$, where the infimum is taken over all such factorizations. We denote by $\mathcal{P} \mathcal{I}(E, F)$ the Banach space of all P-integral operators from $E$ to $F$ endowed with the P-integral norm. We refer to $[6,11]$ for a complete study of these operators.

Observe that if $E$ and $F$ are Banach spaces and $f: E \rightarrow F$ is a linear P-integral operator, then $f$ is Lipschitz P-integral and $\operatorname{Lip}_{P I}(f) \leq\|f\|_{P I}$.

An analogous result to Theorem 2.3 is stated next for Lipschitz P-integral operators with a similar proof.

Theorem 3.1. A Lipschitz operator $f \in \operatorname{Lip}_{0}(X, E)$ is a Lipschitz P-integral operator if and only if there exist a positive regular Borel measure $\nu$ on the Borel $\sigma$-algebra of $B_{X \#}$ with the weak* topology and an operator $\widetilde{A} \in \mathcal{L}\left(L_{1}(\nu), E\right)$ such that $f$ admits the following factorization:

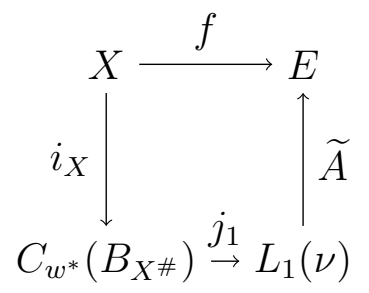

Moreover, $\operatorname{Lip}_{P I}(f)=\inf \|\widetilde{A}\| \nu\left(B_{X \#}\right)$, where the infimum is taken over all such possible $\nu$ 's and $\widetilde{A}$ 's, and this infimum is attained.

We can prove that $\left(\operatorname{Lip}_{0 P I}(X, E), \operatorname{Lip}_{P I}\right)$ is a Banach space and a Banach ideal of Lipschitz operators with a similar proof to that of Proposition 2.1.

Proposition 3.2. $\operatorname{Lip}_{0 P I}(X, E)$ is a linear subspace of $\operatorname{Lip}_{0 G I}(X, E)$ having the following properties:

(i) $\operatorname{Lip}_{G I}(f) \leq \operatorname{Lip}_{P I}(f)$ for all $f \in \operatorname{Lip}_{0 P I}(X, E)$.

(ii) $\operatorname{Lip}_{P I}$ is a Banach space norm on $\operatorname{Lip}_{0 P I}(X, E)$.

(iii) If $g \in X^{\#}$ and $e \in E$, then $g \cdot e \in \operatorname{Lip}_{0 P I}(X, E)$ and $\operatorname{Lip}_{P I}(g \cdot e)=$ $\operatorname{Lip}(g)\|e\|$.

(iv) Let $Y$ be a pointed metric space and $F$ a Banach space. If $h \in \operatorname{Lip}_{0}(Y, X)$, $f \in \operatorname{Lip}_{0 P I}(X, E)$ and $T \in \mathcal{L}(E, F)$, then $T f h \in \operatorname{Lip}_{0 P I}(Y, F)$ and $\operatorname{Lip}_{P I}(T f h) \leq\|T\| \operatorname{Lip}_{P I}(f) \operatorname{Lip}(h)$.

A case in which Lipschitz G-integral operators are Lipschitz P-integral is the following. 
Proposition 3.3. If $\kappa_{E}(E)$ is complemented in $E^{* *}$ by a projection of norm one (in particular, if $E$ is a dual Banach space), then every Lipschitz G-integral operator $f: X \rightarrow E$ is Lipschitz P-integral and $\operatorname{Lip}_{P I}(f)=\operatorname{Lip}_{G I}(f)$.

Proof. Let $P$ be a continuous linear projection from $E^{* *}$ onto $\kappa_{E}(E)$ with $\|P\|=$ 1. Hence $P \kappa_{E}(e)=\kappa_{E}(e)$ for all $e \in E$. Let $f \in \operatorname{Lip}_{0 G I}(X, E)$ and take a Lipschitz G-integral factorization

$$
\kappa_{E} f=A I_{\infty, 1} b: X \stackrel{b}{\rightarrow} L_{\infty}(\mu) \stackrel{I_{\infty}, 1}{\rightarrow} L_{1}(\mu) \stackrel{A}{\rightarrow} E^{* *} .
$$

Hence $\kappa_{E} f=P \kappa_{E} f=P A I_{\infty, 1} b$. Taking $C=\kappa_{E}^{-1} P A \in \mathcal{L}\left(L_{1}(\mu), E\right)$, we have the factorization

$$
f=C I_{\infty, 1} b: X \stackrel{b}{\rightarrow} L_{\infty}(\mu) \stackrel{I_{\infty, 1}}{\rightarrow} L_{1}(\mu) \stackrel{C}{\rightarrow} E,
$$

which shows that $f \in \operatorname{Lip}_{0 P I}(X, E)$ and

$$
\operatorname{Lip}_{P I}(f) \leq\|C\| \operatorname{Lip}(b) \mu(\Omega) \leq\|A\| \operatorname{Lip}(b) \mu(\Omega) .
$$

Passing to the infimum we obtain that $\operatorname{Lip}_{P I}(f) \leq \operatorname{Lip}_{G I}(f)$ and the proof is complete.

We now give a version of Proposition 2.4 for Lipschitz P-integral operators.

Proposition 3.4. Let $f \in \operatorname{Lip}_{0}(X, E)$.

(i) If $f \in \operatorname{Lip}_{0 P I}(X, E)$, then $f^{t} \in \mathcal{P I}\left(E^{*}, X^{\#}\right)$ and $\left\|f^{t}\right\|_{P I} \leq \operatorname{Lip}_{P I}(f)$.

(ii) If $T_{f} \in \mathcal{P} \mathcal{I}(\mathcal{F}(X), E)$, then $f \in \operatorname{Lip}_{0 P I}(X, E)$ and $\operatorname{Lip}_{P I}(f) \leq\left\|T_{f}\right\|_{P I}$.

We are now ready to answer the question that motivated this paper.

Theorem 3.5. A Lipschitz operator $f \in \operatorname{Lip}_{0}\left(X, E^{*}\right)$ is Lipschitz G-integral if and only if the associate linear functional $\Gamma(f)$ on $\mathcal{F}(X) \otimes E$ is continuous on $\mathcal{F}(X) \widehat{\otimes}_{\varepsilon} E$. The mapping $f \mapsto \Gamma(f)$ is an isometric isomorphism from $\left(\operatorname{Lip}_{0 G I}\left(X, E^{*}\right), \operatorname{Lip}_{G I}\right)$ onto $\left(\mathcal{F}(X) \widehat{\otimes}_{\varepsilon} E\right)^{*}$.

Proof. Let $f \in \operatorname{Lip}_{0 G I}\left(X, E^{*}\right)$. Then $f \in \operatorname{Lip}_{0 P I}\left(X, E^{*}\right)$ and $\operatorname{Lip}_{P I}(f)=\operatorname{Lip}_{G I}(f)$ by Proposition 3.3. Let $f=A I_{\infty, 1} b$ be a factorization of $f$, where $(\Omega, \Sigma, \mu)$ is a finite measure space, $A \in \mathcal{L}\left(L_{1}(\mu), E^{*}\right)$ and $b \in \operatorname{Lip}_{0}\left(X, L_{\infty}(\mu)\right)$. For all $x, y \in X$ and $e \in E$, we have

$$
\begin{gathered}
\left\langle\Gamma(f), \delta_{x} \otimes e\right\rangle=\langle f(x), e\rangle=\left\langle A I_{\infty, 1} b(x), e\right\rangle=\left\langle\kappa_{E}(e), A I_{\infty, 1} b(x)\right\rangle \\
=\left\langle\Phi\left(\Phi^{-1} A^{*} \kappa_{E}(e)\right), I_{\infty, 1} b(x)\right\rangle=\int_{\Omega} b(x) \Phi^{-1} A^{*} \kappa_{E}(e) d \mu .
\end{gathered}
$$

Hence $\Gamma(f)$ is continuous on $\mathcal{F}(X) \widehat{\otimes}_{\varepsilon} E$ and $\|\Gamma(f)\| \leq\left\|\Phi^{-1} A^{*} \kappa_{E}\right\| \operatorname{Lip}(b) \mu(\Omega) \leq$ $\|A\| \operatorname{Lip}(b) \mu(\Omega)$ by Theorem 1.3. Passing to the infimum we arrive at $\|\Gamma(f)\| \leq$ $\operatorname{Lip}_{P I}(f)$ and so $\|\Gamma(f)\| \leq \operatorname{Lip}_{G I}(f)$.

Conversely, assume that $\Gamma(f)$ is continuous on $\mathcal{F}(X) \widehat{\otimes}_{\varepsilon} E$. By Theorem 1.3, there exist a finite measure space $(\Omega, \Sigma, \mu)$ and operators $A \in \mathcal{L}\left(E, L_{\infty}(\mu)\right)$ and $b \in \operatorname{Lip}_{0}\left(X, L_{\infty}(\mu)\right)$ such that

$$
\left\langle\Gamma(f), \delta_{x} \otimes e\right\rangle=\int_{\Omega} b(x) A(e) d \mu
$$


for all $x \in X$ and $e \in E$, with $\|\Gamma(f)\|=\|A\| \operatorname{Lip}(b) \mu(\Omega)$. Hence

$$
\begin{gathered}
\langle f(x), e\rangle=\left\langle\Gamma(f), \delta_{x} \otimes e\right\rangle=\int_{\Omega} b(x) A(e) d \mu=\int_{\Omega} I_{\infty, 1} A(e) I_{\infty, 1} b(x) d \mu \\
=\left\langle\Phi(A(e)), I_{\infty, 1} b(x)\right\rangle=\left\langle\kappa_{L_{1}(\mu)}\left(I_{\infty, 1} b(x)\right), \Phi(A(e))\right\rangle=\left\langle A^{*} \Phi^{*} \kappa_{L_{1}(\mu)} I_{\infty, 1} b(x), e\right\rangle
\end{gathered}
$$

for all $x \in X$ and $e \in E$. Putting $C=A^{*} \Phi^{*} \kappa_{L_{1}(\mu)} \in \mathcal{L}\left(L_{1}(\mu), E^{*}\right)$, we have the factorization

$$
f=C I_{\infty, 1} b: X \stackrel{b}{\rightarrow} L_{\infty}(\mu) \stackrel{I_{\infty, 1}}{\rightarrow} L_{1}(\mu) \stackrel{C}{\rightarrow} E^{*} .
$$

This says us that $f \in \operatorname{Lip}_{0 P I}\left(X, E^{*}\right)$ and

$$
\operatorname{Lip}_{P I}(f) \leq\|C\| \operatorname{Lip}(b) \mu(\Omega) \leq\|A\| \operatorname{Lip}(b) \mu(\Omega)=\|\Gamma(f)\| .
$$

Then, by Proposition $3.2(\mathrm{i}), f \in \operatorname{Lip}_{0 G I}\left(X, E^{*}\right)$ and $\operatorname{Lip}_{G I}(f) \leq\|\Gamma(f)\|$.

Hence the mapping $f \mapsto \Gamma(f)$ from $\left(\operatorname{Lip}_{0 G I}\left(X, E^{*}\right), \operatorname{Lip}_{G I}\right)$ to $\left(\mathcal{F}(X) \widehat{\otimes}_{\varepsilon} E\right)^{*}$ is a linear isometry. To prove that it is surjective, take $\varphi \in\left(\mathcal{F}(X) \widehat{\otimes}_{\varepsilon} E\right)^{*}$. Clearly, $\varphi \in\left(\mathcal{F}(X) \widehat{\otimes}_{\pi} E\right)^{*}$ and, by [10, Theorem 4.1], there is $f \in \operatorname{Lip}_{0}\left(X, E^{*}\right)$ such that $\Gamma(f)=\varphi$. This implies that $f \in \operatorname{Lip}_{0 G I}\left(X, E^{*}\right)$ by above-proved and the proof is complete.

From Theorems 2.2 and 3.5, we deduce immediately the following.

Corollary 3.6. Let $f \in \operatorname{Lip}_{0}(X, E)$. Then $f \in \operatorname{Lip}_{0 G I}(X, E)$ if and only if $\kappa_{E} f \in \operatorname{Lip}_{0 G I}\left(X, E^{* *}\right)$. Furthermore, $\operatorname{Lip}_{G I}(f)=\operatorname{Lip}_{G I}\left(\kappa_{E} f\right)$.

\section{LIPSCHITZ NUCLEAR OPERATORS}

Let us recall that a linear operator between Banach spaces $T: E \rightarrow F$ is called nuclear if it has a representation in the form $T=\sum_{n=1}^{\infty} f_{n} \cdot y_{n}$, where $\left\{f_{n}\right\}_{n \in \mathbb{N}}$ and $\left\{y_{n}\right\}_{n \in \mathbb{N}}$ are bounded sequences in $E^{*}, F$ respectively such that $\sum_{n=1}^{\infty}\left\|f_{n}\right\|\left\|y_{n}\right\|<$ $\infty$. The nuclear norm of $T$ is

$$
\|T\|_{N}=\inf \sum_{n=1}^{\infty}\left\|f_{n}\right\|\left\|y_{n}\right\|,
$$

where the infimum is taken over all such representations of $T$. We denote by $\mathcal{N}(E, F)$ the Banach space of all nuclear linear operators from $E$ to $F$ equipped with the nuclear norm. We direct the reader to $[6,11]$ for a study of these operators.

Next we introduce a Lipschitz version of that class of linear operators.

Definition 4. Let $X$ be a pointed metric space and let $E$ be a Banach space. A map $f \in \operatorname{Lip}_{0}(X, E)$ is called a Lipschitz nuclear operator if there exist bounded sequences $\left\{g_{n}\right\}_{n \in \mathbb{N}}$ in $X^{\#}$ and $\left\{e_{n}\right\}_{n \in \mathbb{N}}$ in $E$ such that $f=\sum_{n=1}^{\infty} g_{n} \cdot e_{n}$ satisfying $\sum_{n=1}^{\infty} \operatorname{Lip}\left(g_{n}\right)\left\|e_{n}\right\|<\infty$. The pair $\left(\left\{g_{n}\right\}_{n \in \mathbb{N}},\left\{e_{n}\right\}_{n \in \mathbb{N}}\right)$ is called a Lipschitz nuclear representation of $f$. The Lipschitz nuclear norm of $f$ is

$$
\operatorname{Lip}_{N}(f)=\inf \sum_{n=1}^{\infty} \operatorname{Lip}\left(g_{n}\right)\left\|e_{n}\right\|,
$$

the infimum being taken over all Lipschitz nuclear representations of $f$. The set of all Lipschitz nuclear operators from $X$ to $E$ will be denoted by $\operatorname{Lip}_{0 N}(X, E)$. 
Observe that if $E$ and $F$ are Banach spaces and $f: E \rightarrow F$ is a linear nuclear operator, then $f$ is Lipschitz nuclear and $\operatorname{Lip}_{N}(f) \leq\|f\|_{N}$.

Chen and Zheng introduced in [5] the spaces of strongly Lipschitz $p$-nuclear operators from $X$ to $E$ with the so-called strongly Lipschitz $p$-nuclear norm $s \nu_{p}^{L}$ with $p \in[1, \infty]$. It can be checked easily that every strongly Lipschitz 1-nuclear operator $f: X \rightarrow E$ is Lipschitz nuclear and $\operatorname{Lip}_{N}(f) \leq s \nu_{1}^{L}(f)$. Hence we are considering here a bigger space of Lipschitz operators under a different norm.

We now show that $\left(\operatorname{Lip}_{0 N}(X, E), \operatorname{Lip}_{N}\right)$ is both a Banach space and a Banach ideal of Lipschitz operators.

Proposition 4.1. $\operatorname{Lip}_{0 N}(X, E)$ is a linear subspace of $\operatorname{Lip}_{0}(X, E)$ having the following properties:

(i) $\operatorname{Lip}(f) \leq \operatorname{Lip}_{N}(f)$ for all $f \in \operatorname{Lip}_{0 N}(X, E)$.

(ii) $\operatorname{Lip}_{N}$ is a Banach space norm on $\operatorname{Lip}_{0 N}(X, E)$.

(iii) If $g \in X^{\#}$ and $e \in E$, then $g \cdot e \in \operatorname{Lip}_{0 N}(X, E)$ and $\operatorname{Lip}_{N}(g \cdot \phi)=$ $\operatorname{Lip}(g)\|e\|$.

(iv) Let $Y$ be a pointed metric space and $F$ a Banach space. If $h \in \operatorname{Lip}_{0}(Y, X)$, $f \in \operatorname{Lip}_{0 N}(X, E)$ and $T \in \mathcal{L}(E, F)$, then $T f h \in \operatorname{Lip}_{0 N}(Y, F)$ and

$$
\operatorname{Lip}_{N}(T f h) \leq\|T\| \operatorname{Lip}_{N}(f) \operatorname{Lip}(h) .
$$

Proof. Let $f \in \operatorname{Lip}_{0 N}(X, E)$ and let $f=\sum_{n=1}^{\infty} g_{n} \cdot e_{n}$ be a Lipschitz nuclear representation. Then $f(0)=0$ and, for any $x, y \in X$, we have

$$
\|f(x)-f(y)\|=\left\|\sum_{n=1}^{\infty}\left(g_{n}(x)-g_{n}(y)\right) e_{n}\right\| \leq d(x, y) \sum_{n=1}^{\infty} \operatorname{Lip}\left(g_{n}\right)\left\|e_{n}\right\|,
$$

which implies that $f \in \operatorname{Lip}_{0}(X, E)$ and $\operatorname{Lip}(f) \leq \sum_{n=1}^{\infty} \operatorname{Lip}\left(g_{n}\right)\left\|e_{n}\right\|$. Since $\sum_{n \in \mathbb{N}} g_{n} \cdot e_{n}$ was an arbitrary Lipschitz nuclear representation of $f$, we infer that $\operatorname{Lip}(f) \leq \operatorname{Lip}_{N}(f)$ and this proves $(\mathrm{i})$.

In order to prove that $\operatorname{Lip}_{0 N}(X, E)$ is a linear subspace of $\operatorname{Lip}_{0}(X, E)$, let $\lambda \in \mathbb{K}$. Since $\lambda f=\sum_{n=1}^{\infty}\left(\lambda g_{n}\right) \cdot e_{n}$ and $\sum_{n=1}^{\infty} \operatorname{Lip}\left(\lambda g_{n}\right)\left\|e_{n}\right\|=|\lambda| \sum_{n=1}^{\infty} \operatorname{Lip}\left(g_{n}\right)\left\|e_{n}\right\|$, we deduce that $\lambda f \in \operatorname{Lip}_{0 N}(X, E)$ and $\operatorname{Lip}_{N}(\lambda f) \leq|\lambda| \sum_{n=1}^{\infty} \operatorname{Lip}\left(g_{n}\right)\left\|e_{n}\right\|$. If $\lambda=0$, it follows that $\operatorname{Lip}_{N}(\lambda f)=|\lambda| \operatorname{Lip}_{N}(f)$, and if $\lambda \neq 0$, we have $\operatorname{Lip}_{N}(\lambda f) \leq$ $|\lambda| \operatorname{Lip}_{N}(f)$ which gives $\operatorname{Lip}_{N}(f) \leq|\lambda|^{-1} \operatorname{Lip}_{N}(\lambda f)$ and $|\lambda| \operatorname{Lip}_{N}(f) \leq \operatorname{Lip}_{N}(\lambda f)$. In any case, $\operatorname{Lip}_{N}(\lambda f)=|\lambda| \operatorname{Lip}_{N}(f)$.

Take now $k \in \operatorname{Lip}_{0 N}(X, E)$ and let $\sum_{n \in \mathbb{N}} g_{n}^{\prime} \cdot e_{n}^{\prime}$ be a Lipschitz nuclear representation for $k$. For each $n \in \mathbb{N}$, define

$$
g_{n}^{\prime \prime}=\left\{\begin{array}{ll}
g_{k} & \text { if } \quad n=2 k-1, \\
g_{k}^{\prime} & \text { if } \quad n=2 k .
\end{array} \quad e_{n}^{\prime \prime}=\left\{\begin{array}{lll}
e_{k} & \text { if } & n=2 k-1, \\
e_{k}^{\prime} & \text { if } & n=2 k .
\end{array}\right.\right.
$$

An easy verification shows that $f+k=\sum_{n=1}^{\infty} g_{n}^{\prime \prime} \cdot e_{n}^{\prime \prime}$ and

$$
\sum_{n=1}^{\infty} \operatorname{Lip}\left(g_{n}^{\prime \prime}\right)\left\|e_{n}^{\prime \prime}\right\|=\sum_{n=1}^{\infty} \operatorname{Lip}\left(g_{n}\right)\left\|e_{n}\right\|+\sum_{n=1}^{\infty} \operatorname{Lip}\left(g_{n}^{\prime}\right)\left\|e_{n}^{\prime}\right\| \text {. }
$$


Hence $f+k \in \operatorname{Lip}_{0 N}(X, E)$ and

$$
\operatorname{Lip}_{N}(f+k) \leq \sum_{n=1}^{\infty} \operatorname{Lip}\left(g_{n}\right)\left\|e_{n}\right\|+\sum_{n=1}^{\infty} \operatorname{Lip}\left(g_{n}^{\prime}\right)\left\|e_{n}^{\prime}\right\| .
$$

Passing to the infimum we arrive at $\operatorname{Lip}_{N}(f+k) \leq \operatorname{Lip}_{N}(f)+\operatorname{Lip}_{N}(k)$.

We have just proved that $\operatorname{Lip}_{N}$ is a norm on $\operatorname{Lip}_{0 N}(X, E)$. To show its completeness, let $\left\{f_{n}\right\}_{n \in \mathbb{N}}$ be a sequence in $\operatorname{Lip}_{0 N}(X, E)$ such that $\sum_{n=1}^{\infty} \operatorname{Lip}_{N}\left(f_{n}\right)<\infty$. Then $\sum_{n=1}^{\infty} \operatorname{Lip}\left(f_{n}\right)<\infty$ too, and so $f=\sum_{n=1}^{\infty} f_{n}$ exists in $\operatorname{Lip}_{0}(X, E)$. We will show that $f \in \operatorname{Lip}_{0 N}(X, E)$ and $\operatorname{Lip}_{N}(f) \leq \sum_{n=1}^{\infty} \operatorname{Lip}_{N}\left(f_{n}\right)$. Let $\varepsilon>0$. For each $n \in \mathbb{N}$ choose bounded sequences $\left\{g_{(n, k)}\right\}_{k \in \mathbb{N}}$ in $X^{\#}$ and $\left\{e_{(n, k)}\right\}_{k \in \mathbb{N}}$ in $E$ such that $f_{n}=\sum_{k=1}^{\infty} g_{(n, k)} \cdot e_{(n, k)}$ and $\sum_{k=1}^{\infty} \operatorname{Lip}\left(g_{(n, k)}\right)\left\|e_{(n, k)}\right\|<\operatorname{Lip}_{N}\left(f_{n}\right)+\varepsilon / 2^{n}$. If $\sigma$ is a bijection from $\mathbb{N}$ to $\mathbb{N} \times \mathbb{N}$, we have

$$
f=\sum_{n=1}^{\infty} f_{n}=\sum_{n=1}^{\infty} \sum_{k=1}^{\infty} g_{(n, k)} \cdot e_{(n, k)}=\sum_{m=1}^{\infty} g_{\sigma(m)} \cdot e_{\sigma(m)}
$$

with

$$
\sum_{m=1}^{\infty} \operatorname{Lip}\left(g_{\sigma(m)}\right)\left\|e_{\sigma(m)}\right\|<\sum_{n=1}^{\infty} \operatorname{Lip}_{N}\left(f_{n}\right)+\varepsilon .
$$

So $f \in \operatorname{Lip}_{0 N}(X, E)$ and $\operatorname{Lip}_{N}(f) \leq \sum_{n=1}^{\infty} \operatorname{Lip}_{N}\left(f_{n}\right)$. We can apply the same reasoning to $f-\sum_{k=1}^{n} f_{k}$ to get $\operatorname{Lip}_{N}\left(f-\sum_{k=1}^{n} f_{k}\right) \leq \sum_{k=n+1}^{\infty} \operatorname{Lip}_{N}\left(f_{k}\right)$ for all $n \in \mathbb{N}$, and so $f=\sum_{n=1}^{\infty} f_{n}$ in $\operatorname{Lip}_{0 N}(X, E)$. This completes the proof of (ii).

In order to prove (iii), take $g \in X^{\#}$ and $e \in E$. Clearly, $g \cdot e \in \operatorname{Lip}_{0 N}(X, E)$ and $\operatorname{Lip}_{N}(g \cdot e) \leq \operatorname{Lip}(g)\|e\|$. The reverse inequality is deduced from (i) and Proposition 1.1 (ii).

Finally, we prove (iv). Let $h \in \operatorname{Lip}_{0}(Y, X), f \in \operatorname{Lip}_{0 N}(X, E)$ and $T \in \mathcal{L}(E, F)$. Let $\sum_{n \in \mathbb{N}} g_{n} \cdot e_{n}$ be a Lipschitz nuclear representation for $f$. A simple computation shows that $T f h=\sum_{n=1}^{\infty}\left(g_{n} h\right) \cdot T\left(e_{n}\right)$ where $\left\{g_{n} h\right\}_{n \in \mathbb{N}}$ and $\left\{T\left(e_{n}\right)\right\}_{n \in \mathbb{N}}$ are bounded sequences in $Y^{\#}$ and $F$, respectively. Moreover,

$$
\sum_{n=1}^{\infty} \operatorname{Lip}\left(g_{n} h\right)\left\|T\left(e_{n}\right)\right\| \leq \operatorname{Lip}(h)\|T\| \sum_{n=1}^{\infty} \operatorname{Lip}\left(g_{n}\right)\left\|e_{n}\right\|<\infty .
$$

Therefore $T f h \in \operatorname{Lip}_{0 N}(Y, F)$ and $\operatorname{Lip}_{N}(T f h) \leq \operatorname{Lip}(h)\|T\| \sum_{n=1}^{\infty} \operatorname{Lip}\left(g_{n}\right)\left\|e_{n}\right\|$. This ensures that $\operatorname{Lip}_{N}(T f h) \leq\|T\| \operatorname{Lip}_{N}(f) \operatorname{Lip}(h)$.

Clearly, every Lipschitz finite-rank operator is Lipschitz nuclear. We give the following converse. Let us recall also that a map $f \in \operatorname{Lip}_{0}(X, E)$ is Lipschitz compact if

$$
\left\{\frac{f(x)-f(y)}{d(x, y)}: x, y \in X, x \neq y\right\}
$$

is a relatively compact subset of $E$ (see [9, Definition 2.1]).

Proposition 4.2. Every Lipschitz nuclear operator from $X$ to $E$ is the $\operatorname{Lip}_{N}$-limit of a sequence of Lipschitz finite-rank operators from $X$ to $E$, and so is Lipschitz compact. 
Proof. Let $f \in \operatorname{Lip}_{0 N}(X, E)$ and let $f=\sum_{n=1}^{\infty} g_{n} \cdot e_{n}$ be a Lipschitz nuclear representation. For each $n \in \mathbb{N}$, define $s_{n}=\sum_{k=1}^{n} g_{k} \cdot e_{k}$. Then $\left\{s_{n}\right\}_{n \in \mathbb{N}}$ is a sequence in $\operatorname{Lip}_{0 F}(X, E)$ by Proposition 1.1 and it converges to $f$ in the $\operatorname{Lip}_{N^{-}}$ norm since $\operatorname{Lip}_{N}\left(f-s_{n}\right) \leq \sum_{k=n+1}^{\infty} \operatorname{Lip}\left(g_{k}\right)\left\|e_{k}\right\|$ for all $n \in \mathbb{N}$. Then $\left\{s_{n}\right\}_{n \in \mathbb{N}}$ also converges to $f$ in the Lip-norm by Proposition 4.1 (i), and therefore $f$ is Lipschitz compact. Indeed, since $\operatorname{Lip}\left(s_{n}-f\right)=\left\|T_{s_{n}}-T_{f}\right\|$ and $T_{s_{n}}$ is a finite-rank bounded linear operator from $\mathcal{F}(X)$ to $E$ by [9, Theorem 1.2 and Proposition 2.4], it follows that $T_{f}$ is a compact linear operator from $\mathcal{F}(X)$ to $E$ and then, by [9, Proposition 2.1], $f$ is Lipschitz compact.

We now see that the transpose Lipschitz operator of a Lipschitz nuclear operator is nuclear.

Proposition 4.3. If $f \in \operatorname{Lip}_{0 N}(X, E)$, then $f^{t} \in \mathcal{N}\left(E^{*}, X^{\#}\right)$ and $\left\|f^{t}\right\|_{N} \leq$ $\operatorname{Lip}_{N}(f)$.

Proof. Let $f \in \operatorname{Lip}_{0 N}(X, E)$ and take $\sum_{n \in \mathbb{N}} g_{n} \cdot e_{n}$, a Lipschitz nuclear representation for $f$. Using that the map $f \mapsto f^{t}$ is a continuous linear operator from $\operatorname{Lip}_{0}(X, E)$ to $\mathcal{L}\left(E^{*}, X^{\#}\right)$, we obtain that

$$
f^{t}=\sum_{n=1}^{\infty}\left(g_{n} \cdot e_{n}\right)^{t}=\sum_{n=1}^{\infty} \kappa_{E}\left(e_{n}\right) \cdot g_{n} .
$$

This implies that $f^{t} \in \mathcal{N}\left(E^{*}, X^{\#}\right)$ with $\left\|f^{t}\right\|_{N} \leq \sum_{n=1}^{\infty}\left\|\kappa_{E}\left(e_{n}\right)\right\| \operatorname{Lip}\left(g_{n}\right)=$ $\sum_{n=1}^{\infty}\left\|e_{n}\right\| \operatorname{Lip}\left(g_{n}\right)$, from which $\left\|f^{t}\right\|_{N} \leq \operatorname{Lip}_{N}(f)$.

The three following results unit the work of Section 3 with the current study.

Proposition 4.4. Every Lipschitz nuclear operator $f$ from $X$ to $E$ is Lipschitz $P$-integral and $\operatorname{Lip}_{P I}(f) \leq \operatorname{Lip}_{N}(f)$.

Proof. Suppose that $f=\sum_{n=1}^{\infty} g_{n} \cdot e_{n}$, where $\left\{g_{n}\right\}_{n \in \mathbb{N}}$ and $\left\{e_{n}\right\}_{n \in \mathbb{N}}$ are bounded sequences in $X^{\#}$ and $E$ respectively satisfying $\sum_{n=1}^{\infty} \operatorname{Lip}\left(g_{n}\right)\left\|e_{n}\right\|<\infty$. Let $\Sigma$ be the Borel $\sigma$-algebra of $B_{X \#}$ with its weak* topology and define $\mu: \Sigma \rightarrow E$ by

$$
\mu(G)=\sum_{n=1}^{\infty} \operatorname{Lip}\left(g_{n}\right) \delta_{g_{n} / \operatorname{Lip}\left(g_{n}\right)}(G) e_{n},
$$

where $\delta_{g}$ denotes the point mass concentrated at $g$. It is easy to check that $\mu$ is a vector measure with variation norm $\|\mu\|_{1}:=|\mu|_{1}\left(B_{X^{\#}}\right) \leq \sum_{n=1}^{\infty} \operatorname{Lip}\left(g_{n}\right)\left\|e_{n}\right\|$. Moreover, for all $x \in X$, we have

$$
f(x)=\int_{B_{X} \#} g(x) d \mu(g) .
$$

Define the bounded linear operator $V: C_{w^{*}}\left(B_{X^{\#}}\right) \rightarrow E$ by

$$
V(h)=\int_{B_{X \#}} h(g) d \mu(g) .
$$

Since $\mu$ is of bounded variation, then $V$ is a P-integral operator of $C_{w^{*}}\left(B_{X^{\#}}\right)$ into $E$ with $\|V\|_{P I}=\|\mu\|_{1}$ by [11, Proposition 5.28]. Hence $V$ is a Lipschitz P-integral 
operator with $\operatorname{Lip}_{P I}(V) \leq\|V\|_{P I}$. Now, if $i_{X}$ is the natural injection of $X$ into $C_{w^{*}}\left(B_{X^{\#}}\right)$, we have

$$
V i_{X}(x)=\int_{B_{X} \#} g(x) d \mu(g)=f(x)
$$

for all $x \in X$. Then, by Theorem 3.1 and Proposition 3.2 (iv), $f \in \operatorname{Lip}_{0 P I}(X, E)$ and $\operatorname{Lip}_{P I}(f) \leq \operatorname{Lip}_{P I}(V) \leq\|\mu\|_{1} \leq \sum_{n=1}^{\infty} \operatorname{Lip}\left(g_{n}\right)\left\|e_{n}\right\|$. Passing to the infimum we arrive at $\operatorname{Lip}_{P I}(f) \leq \operatorname{Lip}_{N}(f)$.

A converse of Proposition 4.4 is the following.

Proposition 4.5. Let $X$ be a pointed metric space and $E$ a Banach space. Suppose that $E$ has the Radon-Nikodým property. Then every Lipschitz P-integral operator $f$ from $X$ to $E$ is Lipschitz nuclear and $\operatorname{Lip}_{N}(f) \leq \operatorname{Lip}_{P I}(f)$.

Proof. Let $f \in \operatorname{Lip}_{0 P I}(X, E)$. By Theorem 3.1, there exist a positive regular Borel measure $\nu$ on the Borel $\sigma$-algebra of $B_{X^{\#}}$ with the weak* topology and an operator $\widetilde{A} \in \mathcal{L}\left(L_{1}(\nu), E\right)$ with $\operatorname{Lip}_{P I}(f)=\|\widetilde{A}\| \nu\left(B_{X^{\#}}\right)$ such that

$$
f=\widetilde{A} j_{1} i_{X}: X \stackrel{i_{X}}{\rightarrow} C_{w^{*}}\left(B_{X^{\#}}\right) \stackrel{j_{1}}{\rightarrow} L_{1}(\nu) \stackrel{\widetilde{A}}{\rightarrow} E .
$$

Now take the canonical map $j_{\infty}: C_{w^{*}}\left(B_{X^{\#}}\right) \rightarrow L_{\infty}(\nu)$ and factor $j_{1}$ in the form

$$
j_{1}=I_{\infty, 1} j_{\infty}: C_{w^{*}}\left(B_{X \#}\right) \stackrel{j_{\infty}}{\rightarrow} L_{\infty}(\nu) \stackrel{I_{\infty}, 1}{\rightarrow} L_{1}(\nu) .
$$

Put $U=\widetilde{A} I_{\infty, 1} j_{\infty}: C_{w^{*}}\left(B_{X^{\#}}\right) \rightarrow E$. Clearly, $U$ is a P-integral operator and $\|U\|_{P I} \leq\|\widetilde{A}\| \nu\left(B_{\left.X^{\#}\right)}\right.$. Since $E$ has the Radon-Nikodým property, then $U$ is a nuclear operator and $\|U\|_{N}=\|U\|_{P I}$ by [11, Theorem 5.32]. Hence $U$ is also Lipschitz nuclear and $\operatorname{Lip}_{N}(U) \leq\|U\|_{N}$. Since $f=U i_{X}$, we conclude that $f \in \operatorname{Lip}_{0 N}(X, E)$ with $\operatorname{Lip}_{N}(f) \leq \operatorname{Lip}_{N}(U)$ by Proposition 4.1 (iv). Moreover, $\operatorname{Lip}_{N}(f) \leq \operatorname{Lip}_{P I}(f)$.

When $E$ is a Banach space such that $E^{*}$ has the Radon-Nikodým property, we can identify the space of Lipschitz nuclear operators from $X$ to $E^{*}$ with the dual of $\mathcal{F}(X) \widehat{\otimes}_{\varepsilon} E$.

Corollary 4.6. Let $X$ be a pointed metric space and $E$ a Banach space. Suppose that $E^{*}$ has the Radon-Nikodym property. Then a Lipschitz operator $f \in$ $\operatorname{Lip}_{0}\left(X, E^{*}\right)$ is a Lipschitz nuclear operator if and only if the associate linear functional $\Gamma(f)$ on $\mathcal{F}(X) \otimes E$ is continuous on $\mathcal{F}(X) \widehat{\otimes}_{\varepsilon} E$. The mapping $f \mapsto \Gamma(f)$ is an isometric isomorphism from $\left(\operatorname{Lip}_{0 N}\left(X, E^{*}\right), \operatorname{Lip}_{N}\right)$ onto $\left(\mathcal{F}(X) \widehat{\otimes}_{\varepsilon} E\right)^{*}$.

Proof. Propositions 4.5 and 3.3 provide that $\operatorname{Lip}_{0 N}\left(X, E^{*}\right)=\operatorname{Lip}_{0 P I}\left(X, E^{*}\right)=$ $\operatorname{Lip}_{0 G I}\left(X, E^{*}\right)$ and $\operatorname{Lip}_{N}(f)=\operatorname{Lip}_{P I}(f)=\operatorname{Lip}_{G I}(f)$ for all $f \in \operatorname{Lip}_{0 N}\left(X, E^{*}\right)$. Then the corollary follows by applying Theorem 3.5.

Our ultimate goal is to characterize Lipschitz G-integral operators as Lipschitz weak operator limits of $\operatorname{Lip}_{N}$-bounded nets of Lipschitz nuclear operators. 
Definition 5. The Lipschitz weak operator topology (in short, LWOT) on the space $\operatorname{Lip}_{0}(X, E)$ is the locally convex topology induced by the set of linear functionals:

$$
f \mapsto\langle\phi, f(x)\rangle \quad\left(\phi \in E^{*}, x \in X\right) .
$$

We will need two lemmas. First, it is shown that a LWOT-limit of a $\operatorname{Lip}_{G I^{-}}$ bounded net of Lipschitz G-integral operators is again Lipschitz G-integral.

Lemma 4.7. If $\left\{f_{\gamma}\right\}_{\gamma \in \Gamma}$ is a $\operatorname{Lip}_{G I}$-bounded net of Lipschitz G-integral operators from $X$ to $E$ which converges in the Lipschitz weak operator topology to a Lipschitz operator $f \in \operatorname{Lip}_{0}(X, E)$, then $f$ is Lipschitz G-integral.

Proof. Let $\Gamma\left(\kappa_{E} f\right)$ and $\Gamma\left(\kappa_{E} f_{\gamma}\right)$ be the continuous functionals on $\mathcal{F}(X) \widehat{\otimes}_{\pi} E^{*}$ associated to $f$ and $f_{\gamma}$, respectively. Moreover, since $f_{\gamma} \in \operatorname{Lip}_{0 G I}(X, E)$, then $\Gamma\left(\kappa_{E} f_{\gamma}\right)$ is also continuous on $\mathcal{F}(X) \widehat{\otimes}_{\varepsilon} E^{*}$ with $\operatorname{Lip}_{G I}\left(f_{\gamma}\right)=\left\|\Gamma\left(\kappa_{E} f_{\gamma}\right)\right\|$ by Theorem 2.2.

By hypothesis, $\sup _{\gamma \in \Gamma} \operatorname{Lip}_{G I}\left(f_{\gamma}\right) \leq k$ for some constant $k>0$ and $\left\langle\phi, f_{\gamma}(x)\right\rangle$ converges to $\langle\phi, f(x)\rangle$ for all $\phi \in E^{*}$ and $x \in X$.

Next we check that $\Gamma\left(\kappa_{E} f\right)$ is continuous on $\mathcal{F}(X) \widehat{\otimes}_{\varepsilon} E^{*}$. Let $\sum_{i=1}^{n} \delta_{x_{i}} \otimes \phi_{i} \in$ $\mathcal{F}(X) \widehat{\otimes}_{\varepsilon} E^{*}$. Then, given $\delta>0$, there exists a $\gamma \in \Gamma$ such that

$$
\left|\sum_{i=1}^{n}\left\langle\phi_{i}, f\left(x_{i}\right)\right\rangle\right| \leq\left|\sum_{i=1}^{n}\left\langle\phi_{i}, f_{\gamma}\left(x_{i}\right)\right\rangle\right|+\delta
$$

This means that

$$
\begin{aligned}
\left|\left\langle\Gamma\left(\kappa_{E} f\right), \sum_{i=1}^{n} \delta_{x_{i}} \otimes \phi_{i}\right\rangle\right| & \leq\left|\left\langle\Gamma\left(\kappa_{E} f_{\gamma}\right), \sum_{i=1}^{n} \delta_{x_{i}} \otimes \phi_{i}\right\rangle\right|+\delta \\
& \leq k \varepsilon\left(\sum_{i=1}^{n} \delta_{x_{i}} \otimes \phi_{i}\right)+\delta .
\end{aligned}
$$

By the arbitrariness of $\delta$, it follows that

$$
\left|\left\langle\Gamma\left(\kappa_{E} f\right), \sum_{i=1}^{n} \delta_{x_{i}} \otimes \phi_{i}\right\rangle\right| \leq k \varepsilon\left(\sum_{i=1}^{n} \delta_{x_{i}} \otimes \phi_{i}\right) .
$$

Hence $\Gamma\left(\kappa_{E} f\right)$ is continuous on $\mathcal{F}(X) \widehat{\otimes}_{\varepsilon} E^{*}$. Then $f \in \operatorname{Lip}_{0 G I}(X, E)$ by Theorem 2.2 .

Let us recall that a mapping between Banach spaces $Q: E \rightarrow F$ is a quotient mapping if $Q$ is surjective and $\|f\|=\inf \{\|e\|: e \in E, Q(e)=f\}$ for every $f \in F$.

By [11, Proposition 2.8], if $E$ and $F$ are Banach spaces, then every element $T \in E \widehat{\otimes}_{\pi} F$ admits a representation $T=\sum_{n=1}^{\infty} x_{n} \otimes y_{n}$ where $\left\{x_{n}\right\}_{n \in \mathbb{N}}$ and $\left\{y_{n}\right\}_{n \in \mathbb{N}}$ are bounded sequences in $E, F$ respectively such that $\sum_{n=1}^{\infty}\left\|x_{n}\right\|\|\| y_{n} \|<\infty$. Moreover,

$$
\pi(T)=\inf \left\{\sum_{n=1}^{\infty}\left\|x_{n}\right\|\left\|y_{n}\right\|: \sum_{n=1}^{\infty}\left\|x_{n}\right\|\left\|y_{n}\right\|<\infty, T=\sum_{n=1}^{\infty} x_{n} \otimes y_{n}\right\}
$$


the infimum being taken over all the representations of $T \in E \widehat{\otimes}_{\pi} F$ as described above.

Lemma 4.8. Let $X$ be a pointed metric space and $E$ a Banach space. Let $J: X^{\#} \widehat{\otimes}_{\pi} E \rightarrow \operatorname{Lip}_{0 N}(X, E)$ be the formal identity defined for all pairs of bounded sequences $\left\{g_{n}\right\}_{n \in \mathbb{N}}$ in $X^{\#}$ and $\left\{e_{n}\right\}_{n \in \mathbb{N}}$ in $E$ such that $\sum_{n=1}^{\infty} \operatorname{Lip}\left(g_{n}\right)\left\|e_{n}\right\|<\infty$ as

$$
J\left(\sum_{n=1}^{\infty} g_{n} \otimes e_{n}\right)=\sum_{n=1}^{\infty} g_{n} \cdot e_{n} .
$$

Then $J$ is a well-defined quotient mapping.

Proof. Let $T \in X^{\#} \widehat{\otimes}_{\pi} E$. We claim that $J(T) \in \operatorname{Lip}_{0 N}(X, E)$ is independent of the chosen representation as above for $T$. It is known that $\left(X^{\#} \widehat{\otimes}_{\pi} E\right)^{*}$ is isometrically isomorphic to $\mathcal{L}\left(X^{\#}, E^{*}\right)$, with the duality pairing given by

$$
\left\langle S, \sum_{n=1}^{\infty} g_{n} \otimes e_{n}\right\rangle=\sum_{n=1}^{\infty}\left\langle S\left(g_{n}\right), e_{n}\right\rangle
$$

for $\sum_{n=1}^{\infty} g_{n} \otimes e_{n} \in X^{\#} \widehat{\otimes}_{\pi} E$ and $S \in \mathcal{L}\left(X^{\#}, E^{*}\right)$. If $T=\sum_{n=1}^{\infty} g_{n} \otimes e_{n}=$ $\sum_{n=1}^{\infty} g_{n}^{\prime} \otimes e_{n}^{\prime}$, where $\left\{g_{n}\right\}_{n \in \mathbb{N}}$ and $\left\{g_{n}^{\prime}\right\}_{n \in \mathbb{N}}$ in $X^{\#}$ and $\left\{e_{n}\right\}_{n \in \mathbb{N}}$ and $\left\{e_{n}^{\prime}\right\}_{n \in \mathbb{N}}$ in $E$ are bounded sequences satisfying $\left(\sum_{n=1}^{\infty} \operatorname{Lip}\left(g_{n}\right)\left\|e_{n}\right\|\right)\left(\sum_{n=1}^{\infty} \operatorname{Lip}\left(g_{n}^{\prime}\right)\left\|e_{n}^{\prime}\right\|\right)<\infty$, then $\langle S, T\rangle=\sum_{n=1}^{\infty}\left\langle S\left(g_{n}\right), e_{n}\right\rangle=\sum_{n=1}^{\infty}\left\langle S\left(g_{n}^{\prime}\right), e_{n}^{\prime}\right\rangle$ for all $S \in \mathcal{L}\left(X^{\#}, E^{*}\right)$. In particular, if $x \in X$ and $\phi \in E^{*}$, taking $S_{(x, \phi)}$ in $\mathcal{L}\left(X^{\#}, E^{*}\right)$ defined by

$$
\left\langle S_{(x, \phi)}(g), e\right\rangle=g(x) \phi(e) \quad\left(g \in X^{\#}, e \in E\right),
$$

we obtain that $\sum_{n=1}^{\infty} g_{n}(x) \phi\left(e_{n}\right)=\sum_{n=1}^{\infty} g_{n}^{\prime}(x) \phi\left(e_{n}^{\prime}\right)$ for all $x \in X$ and $\phi \in E^{*}$. From this we deduce that $J(T)=\sum_{n=1}^{\infty} g_{n} \cdot e_{n}=\sum_{n=1}^{\infty} g_{n}^{\prime} \cdot e_{n}^{\prime}$, which proves the claim.

Let $f \in \operatorname{Lip}_{0 N}(X, E)$ and let $\sum_{n=1}^{\infty} g_{n} \cdot e_{n}$ be a Lipschitz nuclear representation of $f$. Take $T=\sum_{n=1}^{\infty} g_{n} \otimes e_{n}$. Clearly, $T \in X^{\#} \widehat{\otimes}_{\pi} E$ and $J(T)=f$. Hence $J$ is surjective and $\pi(T) \leq \sum_{n=1}^{\infty} \operatorname{Lip}\left(g_{n}\right)\left\|e_{n}\right\|$. It follows that $\pi(T) \leq \operatorname{Lip}_{N}(f)$, and so

$$
\inf \left\{\pi(T): T \in X^{\#} \widehat{\otimes}_{\pi} E, J(T)=f\right\} \leq \operatorname{Lip}_{N}(f) .
$$

To get the converse inequality, take $T \in X^{\#} \widehat{\otimes}_{\pi} E$ such that $J(T)=f$. Let $\sum_{n=1}^{\infty} g_{n}^{\prime} \otimes e_{n}^{\prime}$ be an arbitrary representation of $T$ where $\left\{g_{n}^{\prime}\right\}_{n \in \mathbb{N}}$ and $\left\{e_{n}^{\prime}\right\}_{n \in \mathbb{N}}$ are bounded sequences in $X^{\#}$ and $E$ respectively such that $\sum_{n=1}^{\infty} \operatorname{Lip}\left(g_{n}^{\prime}\right)\|\| e_{n}^{\prime} \|<\infty$. Then $\sum_{n=1}^{\infty} g_{n}^{\prime} \cdot e_{n}^{\prime}$ is a Lipschitz nuclear representation for $f$ and thus $\operatorname{Lip}_{N}(f) \leq$ $\sum_{n=1}^{\infty} \operatorname{Lip}\left(g_{n}^{\prime}\right)\left\|e_{n}^{\prime}\right\|$. Hence $\operatorname{Lip}_{N}(f) \leq \pi(T)$ and so

$$
\operatorname{Lip}_{N}(f) \leq \inf \left\{\pi(T): T \in X^{\#} \widehat{\otimes}_{\pi} E, J(T)=f\right\} .
$$

We now are ready to obtain the announced result.

Theorem 4.9. A Lipschitz operator $f \in \operatorname{Lip}_{0}(X, E)$ is a Lipschitz G-integral operator if and only if $f$ is the limit in the Lipschitz weak operator topology on $\operatorname{Lip}_{0}(X, E)$ of a $\operatorname{Lip}_{N}$-bounded net of Lipschitz nuclear operators from $X$ to $E$. 
Proof. Assume that $f$ is the LWOT-limit of a $\operatorname{Lip}_{N}$-bounded net of operators in $\operatorname{Lip}_{0 N}(X, E)$. Then $f$ is the LWOT-limit of a $\operatorname{Lip}_{G I}$-bounded net of operators in $\operatorname{Lip}_{0 G I}(X, E)$ by Propositions 4.4 and 3.2. Hence $f \in \operatorname{Lip}_{0 G I}(X, E)$ by Lemma 4.7.

Conversely, let $f \in \operatorname{Lip}_{0 G I}(X, E)$. Then the linear functional $\Gamma\left(\kappa_{E} f\right)$ defined on $\mathcal{F}(X) \otimes E^{*}$ by

$$
\left\langle\Gamma\left(\kappa_{E} f\right), \delta_{x} \otimes \phi\right\rangle=\langle\phi, f(x)\rangle
$$

is continuous on $\mathcal{F}(X) \widehat{\otimes}_{\varepsilon} E^{*}$. It is not hard that the map $j: \mathcal{F}(X) \otimes_{\varepsilon} E^{*} \rightarrow$ $\left(X^{\#} \widehat{\otimes}_{\pi} E\right)^{*}$, defined by

$$
\left\langle j\left(\sum_{i=1}^{n} \delta_{x_{i}} \otimes \phi_{i}\right), \sum_{k=1}^{\infty} g_{k} \otimes e_{k}\right\rangle=\sum_{k=1}^{\infty} \sum_{i=1}^{n} g_{k}\left(x_{i}\right) \phi_{i}\left(e_{k}\right),
$$

is a linear isometry and therefore so is its extension, denoted also by $j$, to $\mathcal{F}(X) \widehat{\otimes}_{\varepsilon} E^{*}$. Then the adjoint map $j^{*}:\left(X^{\#} \widehat{\otimes}_{\pi} E\right)^{* *} \rightarrow\left(\mathcal{F}(X) \widehat{\otimes}_{\varepsilon} E^{*}\right)^{*}$ is surjective. Therefore there is a $T \in\left(X^{\#} \widehat{\otimes}_{\pi} E\right)^{* *}$ such that $j^{*}(T)=\Gamma\left(\kappa_{E} f\right)$. Denote by $\kappa_{A}$ the canonical injection from $X^{\#} \widehat{\otimes}_{\pi} E$ into its bidual. By Goldstine theorem's there exists a net $\left\{T_{\gamma}\right\}_{\gamma \in \Gamma}$ in $X^{\#} \widehat{\otimes}_{\pi} E$ such that $\sup _{\gamma \in \Gamma} \pi\left(T_{\gamma}\right) \leq\|T\|<\infty$ and $\left\langle\kappa_{A}\left(T_{\gamma}\right), v\right\rangle \rightarrow\langle T, v\rangle$ for all $v \in\left(X^{\#} \widehat{\otimes}_{\pi} E\right)^{*}$. Hence $\left\langle\kappa_{A}\left(T_{\gamma}\right), j(u)\right\rangle \rightarrow\langle T, j(u)\rangle$ for all $u \in \mathcal{F}(X) \widehat{\otimes}_{\varepsilon} E^{*}$. In particular, $\left\langle\kappa_{A}\left(T_{\gamma}\right), j\left(\delta_{x} \otimes \phi\right)\right\rangle \rightarrow\left\langle T, j\left(\delta_{x} \otimes \phi\right)\right\rangle$ for all $x \in X$ and $\phi \in E^{*}$. If $T_{\gamma}=\sum_{k=1}^{\infty} g_{\gamma, k} \otimes e_{\gamma, k}$, where $\left\{g_{\gamma, k}\right\}_{k \in \mathbb{N}}$ and $\left\{e_{\gamma, k}\right\}_{k \in \mathbb{N}}$ are bounded sequences in $X^{\#}, E$ respectively such that $\sum_{k=1}^{\infty} \operatorname{Lip}\left(g_{\gamma, k}\right)\left\|e_{\gamma, k}\right\|<\infty$, notice that

$$
\begin{gathered}
\left\langle\kappa_{A}\left(T_{\gamma}\right), j\left(\delta_{x} \otimes \phi\right)\right\rangle=\left\langle j\left(\delta_{x} \otimes \phi\right), T_{\gamma}\right\rangle=\sum_{k=1}^{\infty} g_{\gamma, k}(x) \phi\left(e_{\gamma, k}\right) \\
=\left\langle\phi, \sum_{k=1}^{\infty} g_{\gamma, k}(x) e_{\gamma, k}\right\rangle=\left\langle\phi, J\left(T_{\gamma}\right)(x)\right\rangle
\end{gathered}
$$

where $J: X^{\#} \widehat{\otimes}_{\pi} E \rightarrow \operatorname{Lip}_{0 N}(X, E)$ is the formal identity given in Lemma 4.8, and also

$$
\left\langle T, j\left(\delta_{x} \otimes \phi\right)\right\rangle=\left\langle j^{*}(T), \delta_{x} \otimes \phi\right\rangle=\left\langle\Gamma\left(\kappa_{E} f\right), \delta_{x} \otimes \phi\right\rangle=\langle\phi, f(x)\rangle .
$$

So $\left\langle\phi, J\left(T_{\gamma}\right)(x)\right\rangle \rightarrow\langle\phi, f(x)\rangle$ for all $x \in X$ and $\phi \in E^{*}$. Since $\left\{J\left(T_{\gamma}\right)\right\}_{\gamma \in \Gamma}$ is a $\operatorname{Lip}_{N^{-}}$-bounded net in $\operatorname{Lip}_{0 N}(X, E)$ by Lemma 4.8, the proof is complete.

Since $\operatorname{Lip}_{0 F}(X, E)$ is $\operatorname{Lip}_{N^{-}}$dense in $\operatorname{Lip}_{0 N}(X, E)$ by Proposition 4.2 , we obtain the next refinement of Theorem 4.9.

Corollary 4.10. A Lipschitz operator $f \in \operatorname{Lip}_{0}(X, E)$ is a Lipschitz G-integral operator if and only if $f$ is the limit in the Lipschitz weak operator topology on $\operatorname{Lip}_{0}(X, E)$ of a $\operatorname{Lip}_{N}$-bounded net of Lipschitz finite-rank operators from $X$ to $E$.

Acknowledgement. The authors thank the referee for the suggestions given. This research was partially supported by Junta de Andalucía grant FQM-194. 


\section{REFERENCES}

1. J.A. Chávez-Domínguez, Duality for Lipschitz p-summing operators, J. Funct. Anal. 261 (2011), no. 2, 387-407.

2. J.A. Chávez-Domínguez, Lipschitz $(q, p)$-mixing operators, Proc. Amer. Math. Soc. 140 (2012), no. 9, 3101-3115.

3. J.A. Chávez-Domínguez, Completely (q,p)-mixing maps, Illinois J. Math. 56 (2012), no. 4, $1169-1183$.

4. D. Chen and B. Zheng, Remarks on Lipschitz p-summing operators, Proc. Amer. Math. Soc. 139 (2011), no. 8, 2891-2898.

5. D. Chen and B. Zheng, Lipschitz p-integral operators and Lipschitz p-nuclear operators, Nonlinear Analysis 75 (2012), no. 13, 5270-5282.

6. J. Diestel, H. Jarchow and A. Tonge, Absolutely summing operators, Cambridge Studies in Advanced Mathematics, 43, Cambridge University Press, Cambridge, 1995.

7. J.D. Farmer and W.B. Johnson, Lipschitz p-summing operators, Proc. Amer. Math. Soc. 137 (2009), no. 9, 2989-2995.

8. G. Godefroy and N.J. Kalton, Lipschitz-free Banach spaces, Studia Math. 159 (2003), no. $1,121-141$.

9. A. Jiménez-Vargas, J.M. Sepulcre and Moisés Villegas-Vallecillos, Lipschitz compact operators, J. Math. Anal. Appl. 415 (2014), no. 2, 889-901.

10. J.A. Johnson, Banach spaces of Lipschitz functions and vector-valued Lipschitz functions, Trans. Amer. Math. Soc. 148 (1970), 147-169.

11. R.A. Ryan, Introduction to Tensor Products of Banach Spaces, Series: Springer Monographs in Mathematics, Springer, 2002.

12. K. Saadi, Some properties of Lipschitz strongly p-summing operators, J. Math. Anal. Appl. 423 (2015), no. 2, 1410-1426.

13. N. Weaver, Lipschitz Algebras, World Scientific Publishing Co., Singapore, 1999.

Departamento de Matemáticas, Universidad de Almería, 04120, Almería, Spain. E-mail address: m_gador@hotmail.com

E-mail address: ajimenez@ual.es 